\title{
FISH: Linux System Calls for FPGA Accelerators
}

by

\author{
Kevin Nam \\ A thesis submitted in conformity with the requirements \\ for the degree of Master of Applied Science
}

Edward S. Rogers Sr. Department of Electrical \& Computer Engineering University of Toronto

Copyright (C) 2017 by Kevin Nam 


\begin{abstract}
FISH: Linux System Calls for FPGA Accelerators

Kevin Nam

Master of Applied Science

Edward S. Rogers Sr. Department of Electrical \& Computer Engineering University of Toronto
\end{abstract}

2017

This work presents the FISH (FPGA-Initiated Software-Handled) framework which allows FPGA accelerators to make system calls to the Linux operating system in CPU-FPGA systems. A special FISH Linux kernel module running on the CPU provides a system call interface for FPGA accelerators, much like the $A B I$ which exists for software programs. The work presents the proposed framework that facilitates the FPGA to Linux kernel system call interface. The work also presents a proof-of-concept implementation of the framework running on an Intel Cyclone V SoC device. The FISH framework could be especially useful for high-level synthesis (HLS) by making it possible to synthesize software code that contains system calls. This would expand the subset of code that is currently synthesizable, and allow behavior in accelerators that was previously not possible.

This work was presented in the 2017 International Conference on Field-Programming Logic in Ghent. The corresponding paper, titled "FISH: Linux System Calls for FPGA Accelerators" is available in the conference proceedings. 


\section{Acknowledgments}

I would like to thank my research supervisor Prof. Stephen Brown for all the opportunities, knowledge, and experience that he has given me over the years.

I would like to thank my colleague Blair Fort for all his input and expertise whenever I had a question about anything.

Lastly I would like to recognize the Intel FPGA University Program for board donations that allowed me to implement my design. 


\section{Table of Contents}

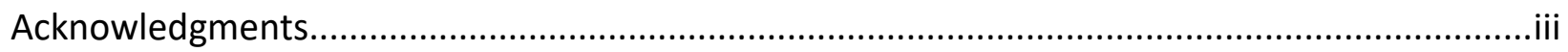

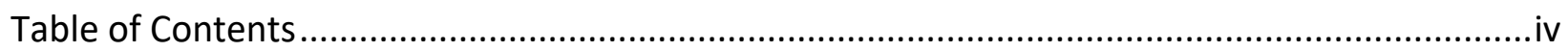



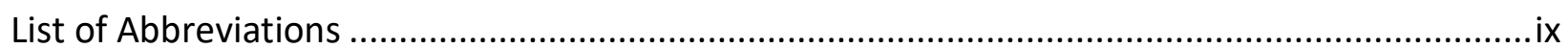

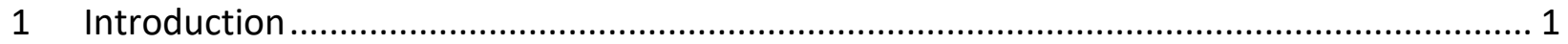

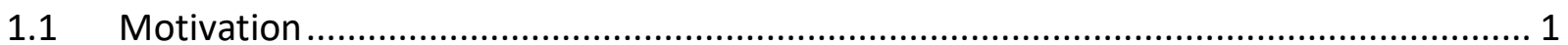

1.2 Contributions



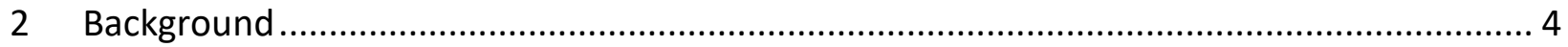

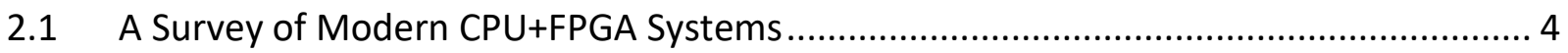

2.1.1 SoC FPGA Devices ………………………....................................................... 4

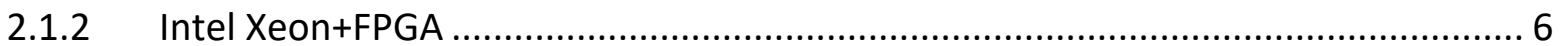

2.1.3 Workstation with PCle FPGA Card ……............................................................... 6

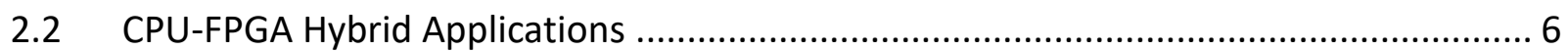

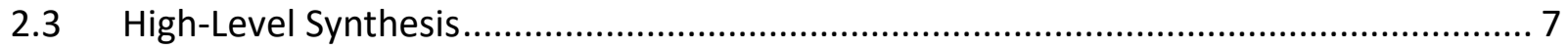

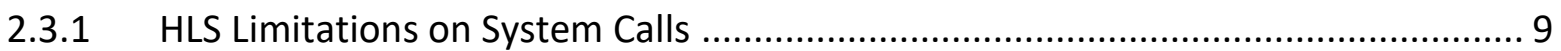

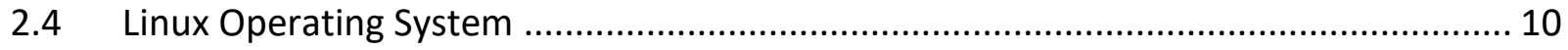

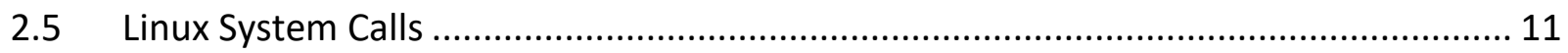


3 FPGA-Initiated Linux System Calls: Potential Use Cases

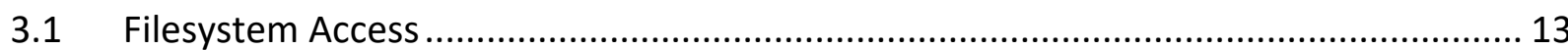

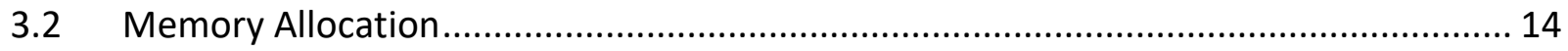



$3.4 \quad$ Thread-Accelerator Synchronization ................................................................. 16

4 The FPGA-Initiated Software-Handled Framework ...................................................... 17

4.1 A Standard System Call Interface for FPGA Accelerators ..................................... 17

4.2 Handling an FPGA-Initiated System Call................................................................. 19

4.3 FPGA to Kernel Module Communication ........................................................ 20

4.4 Handling the System Call Request in the FISH Kernel Module .................................. 22

$4.5 \quad$ Handling System Calls with Persistent Effects .................................................. 23



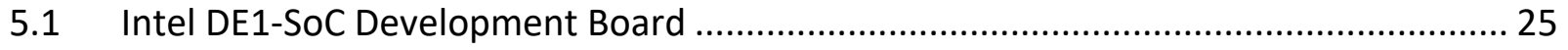

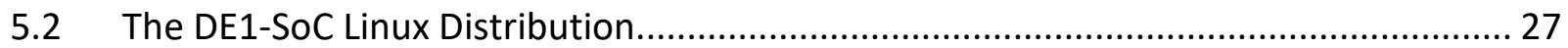

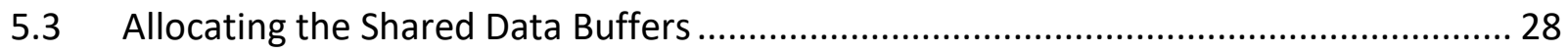

5.4 Implementing the Host Program ..................................................................... 28

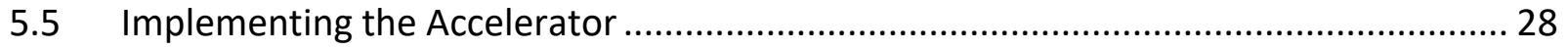

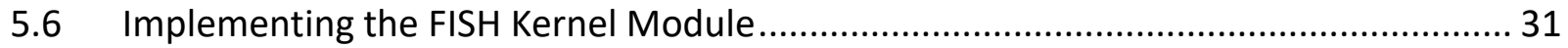

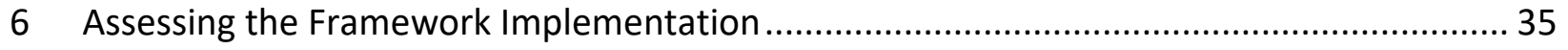




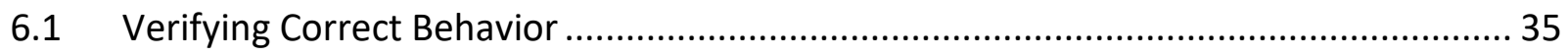





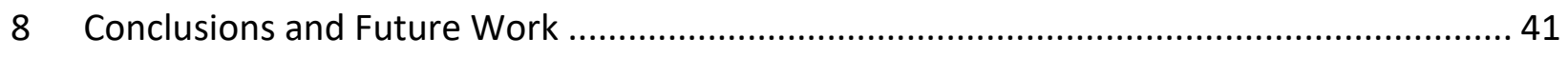

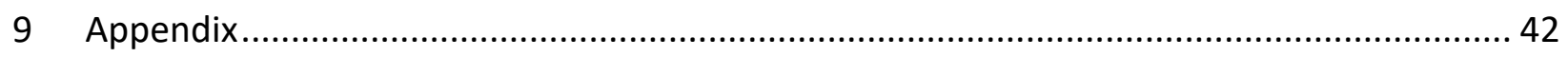

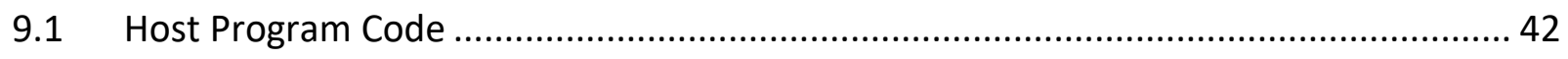



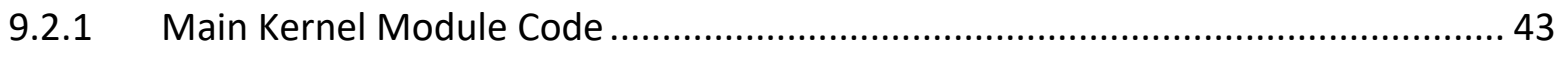

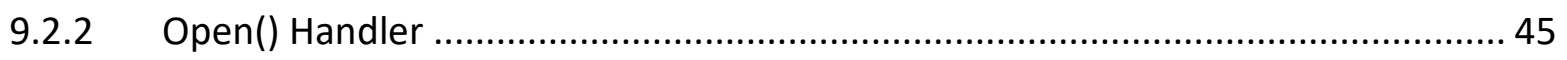

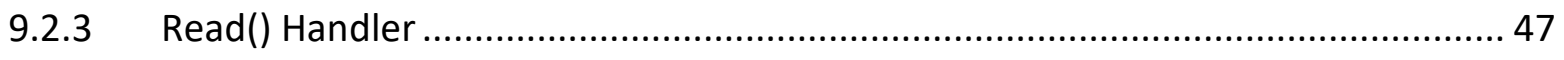

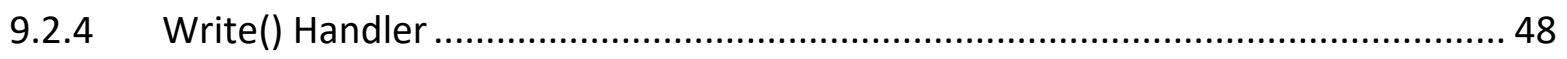



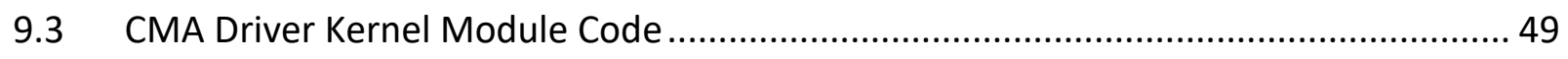

9.4 LegUp C-Language Code for Accelerator ....................................................... 52

9.5 LegUp-Synthesized Accelerator Code in Verilog HDL ......................................... 52

9.6 Verilog HDL Module that Initiates the Open System Call ........................................ 52

9.7 Verilog HDL Module that Initiates the Write System Call ....................................... 56

9.8 Verilog HDL Module that Initiates the Close System Call ........................................6 60






\section{List of Figures}

Figure 1 Example of high level synthesis with C-code as input ............................................. 8

Figure 2 Filesystem accesses from the FPGA accelerator ................................................ 13

Figure 3: Parallels between the traditional system call convention and the FPGA-initiated, SW-

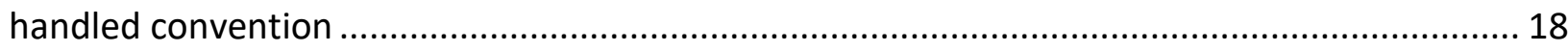

Figure 4: Steps for handling an FPGA-initiated system call ................................................ 19

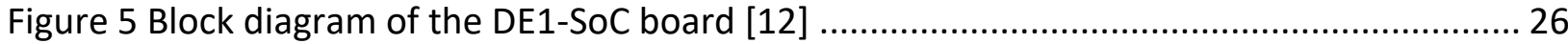

Figure 6: C-language function that was accelerated using LegUp HLS................................... 29

Figure 7 Register interface of the FISH-compatible accelerator ............................................ 30

Figure 8: Registering a threaded IRQ handler ................................................................ 32

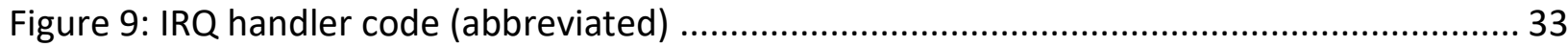

Figure 10: Abbreviated code for the open system call handler ............................................ 34

Figure 11: An accelerator that opens a file and returns the file descriptor ............................. 35

Figure 12: Latencies of Pure-SW and HW-initiated system calls ........................................ 37

Figure $13 \mathrm{C}$-language code for the host program that launches the accelerator ..................... 43

Figure 14 Kernel module code for allocating physically contiguous memory using CMA .......... 51

Figure 15 Accelerator code (in C) that was synthesized using LegUP ....................................5 52

Figure 16 Accelerator code (in Verilog HDL) that was generated by LegUP ........................... 52 
Figure 17 Verilog module that initiates the open() system call from the accelerator ................ 56

Figure 18 Verilog module that initiates the write() system call from the accelerator ................ 59

Figure 19 Verilog module that initiates the close() system call from the accelerator ................ 62

Figure 20 Linux kernel configuration settings for the custom 3.18 kernel used in the DE1-SoC

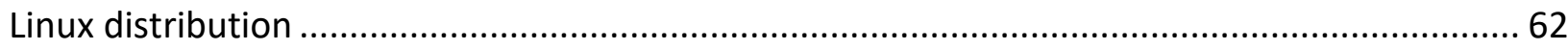




\section{List of Abbreviations}

$\begin{array}{ll}\text { API } & \text { Application Programming Interface } \\ \text { ARM } & \text { Advanced RISC Machines } \\ \text { CMA } & \text { Contiguous Memory Allocator } \\ \text { CPU } & \text { Central Processing Unit } \\ \text { CWD } & \text { Current Working Directory } \\ \text { DMA } & \text { Direct Memory Access } \\ \text { FD } & \text { File Descriptor } \\ \text { FISH } & \text { FPGA-Initiated Software-Handled } \\ \text { FPGA } & \text { Field Programmable Gate Array } \\ \text { FSM } & \text { Finite State Machine } \\ \text { HLS } & \text { High-Level Synthesis } \\ \text { HPS } & \text { Hard Processor System } \\ \text { IRQ } & \text { Interrupt Request } \\ \text { KM } & \text { Kernel Module } \\ \text { LKM } & \text { Linux Kernel Module } \\ \text { OS } & \text { Operating System } \\ \text { PCle } & \text { Peripheral Component Interconnect Express Bus } \\ \text { PID } & \text { Process ID } \\ \text { RAM } & \text { Random Access Memory } \\ \text { RISC } & \text { Reduced Instruction Set Computer } \\ \text { SGDMA } & \text { Scatter-Gather DMA } \\ \text { SoC } & \text { System on Chip } \\ & \end{array}$




\section{Introduction}

\subsection{Motivation}

FPGAs are becoming increasingly integrated into modern day computers. This can be seen across produce ranges of FPGA manufacturers, such as the Intel/Altera SoC [1], the Xilinx SoC/MPSoC [2], and Intel Xeon+FPGA processors [3]. To support the programming of these non-traditional heterogeneous systems, much work has been done in area of CPU+FPGA codesign to provide programming frameworks that encompass the CPU+FPGA system. Popular programming frameworks include the Intel OpenCL SDK [4], Xilinx Vivado High-Level Synthesis tool [5], and LegUP HLS [6]. These frameworks allow the development of hybrid applications, which are applications consisting of "host" software running on the CPU, and accelerator(s) running on the FPGA which work together to provide the application behavior. Hybrid applications are becoming increasingly popular in the datacenter, with cloud service providers such as Amazon AWS and Microsoft Azure adding FPGAs to their datacenters to allow FPGA acceleration of tasks. Often, CPU+FPGA heterogeneous systems run an operating system like Linux. The Linux OS is frequently used as it is free and offers system management tools, greater productivity through access to higher-level APIs and device drivers, and a myriad of freely available Linux software. When a developer creates a hybrid application for a CPU-FPGA system running Linux, there are many considerations involved. One consideration is how to provide the FPGA circuity access to Linux-provided resources and devices. In a purely-software application, the software program running on Linux requests access to these resources via Linux system calls. Unfortunately, the Linux system call interface is only available to software, and a circuit in 
the FPGA is unable to use it. This means that traditionally, FPGA accelerators could not access Linux-provided resources directly, and had to instead rely on the host software to request the resources and relay them to the FPGA accelerator. Not only does the host program intermediary approach add latency, this approach also means that the developer must code the host program with this behavior in mind, adding to development complexity. In a technological landscape where CPU and FPGA are more tightly integrated than ever before, it seems that a way for FPGAs to directly make system calls to the operating system could be a potential benefit to hybrid applications.

\subsection{Contributions}

The objective of this work to provide a standardized interface to FPGA accelerators for making Linux system calls. Key contributions are:

1. Development of an FPGA to operating system interface for making system calls.

2. Proof of concept implementation of the system call interface.

\subsection{Thesis Organization}

The rest of this document is organized as follows:

Section 2 provides background information on current CPU+FPGA systems, current approaches to CPU+FPGA hybrid applications, high-level synthesis, the Linux operating system, and Linux system calls. 
Section 3 presents some possible use cases of a system call interface to FPGAs. It discusses potential uses like filesystem access, memory allocation, device access, and thread synchronization.

Section 4 presents our proposed FPGA-Initiated, Software-Handled (FISH) framework. It describes the system call interface to FPGAs, as well as the framework for handling the system calls on the operating-system side. It outlines and describes the key components of this framework, which include the host program, the FPGA accelerator, and the FISH Linux kernel module.

Section 5 describes our proof of concept implementation of the FISH framework. It goes over key considerations for implementing the framework such as the necessary modifications to existing code, how the FISH kernel module was implemented, and the hardware-architecturespecific details.

Section 5 briefly assesses the performance of our proof of concept implementation, to compare the runtime of the FPGA-to-CPU system calls against the best possible case (CPU-to-CPU system calls).

Section 7 discusses some prior related works, and Section 8 presents concluding remarks and suggestions for future work. 


\section{Background}

\subsection{A Survey of Modern CPU+FPGA Systems}

\subsubsection{SoC FPGA Devices}

Top FPGA vendors Intel and Xilinx offer what are commonly referred to as SoC FPGA devices. Simply put, they are devices that comprise an FPGA and a CPU in the same die with some channels of communication between the two halves. The offerings from both companies are largely similar, and both vendors offer devices that contain an FPGA of varying size and capability, in addition to a single or multicore ARM processor ranging from the ARM Cortex-A9, ARM Cortex-A53, to the ARM Cortex-R5.

Communication between the FPGA and CPU are carried out through hardened communication channels built into the chip. In the case of the Intel Cyclone V SoC, three memory-mapped bridges (connected to AXI-compatible masters/slaves at either end) exist to facilitate communication. Two of the bridges, the HPS2FPGA and the LWHPS2FPGA, facilitate CPU to FPGA communication, while the FPGA2HPS bridge facilitates FPGA to CPU communication. The bridges are hard-wired to interconnect in the CPU-side of the chip, but can be configured on the FPGA-side to connect to any user-defined circuitry capable of AXI communication. Developers use Intel's QSys software to configure the connections on the FPGA side and generate the corresponding HDL for compilation.

In addition to the CPU and the FPGA, vendors place hardened controllers like USB, SDRAM, Ethernet, SDMMC, SPI, and I2C, so developers do not have to use FPGA fabric for these 
commonly used tasks. An important note is that these peripheral devices are generally placed in the CPU half of the chip, allowing direct memory-mapped access to their register interfaces by the CPU. When Linux runs on the SoC device, its built-in drivers for these peripherals allow developers to use higher-level API to access and operate these devices, offering a significant productivity advantage. This is in contrast to having to write $\mathrm{HDL}$ to implement the equivalent peripheral device in the FPGA, and writing FSMs in hardware or baremetal code running on a soft-CPU in the FPGA to control the peripheral device. When writing a Linux hybrid application that uses one of the Linux -controlled devices, the host program (the portion of the application running on the $\mathrm{CPU}$ ) would typically have to facilitate the operations. If the FPGA circuit required one of these devices, the host program would likely have to be the intermediary, potentially adding unwanted latency. Additionally, on the same system, there may be standalone FPGA circuits that require access to one of the Linux-controlled devices. While the SoC FPGA chip offers FPGA-to-CPU communication channel(s) that would allow the standalone circuit to directly access the device, doing so would require synchronization with the Linux OS due to potential contention. In both cases of FPGA circuitry requiring Linux-controlled devices mentioned above, it would be beneficial to have some method by which an FPGA circuit could directly request OS services and devices through system calls. This solves the unnecessary latency added by the host program intermediary, and solves the issue of synchronization with the Linux OS in the case of standalone FPGA circuit access (since it would be the Linux OS that would handle the requests from the FPGA circuit). 


\subsubsection{Intel Xeon+FPGA}

Although not yet available, the Intel Xeon+FPGA device is significant due to the impact it may have on the datacenter market. As of December 2016, Intel Xeon processors had a 90\%+ market share of the world's datacenters [7]. A push by Intel to incorporate FPGA devices into the data center would mean a huge increase overall in the world's usage of FPGA devices and in turn, the prevalence of CPU+FPGA hybrid applications. This would mean an increased demand for CPU-FPGA hybrid application development resources and methods, which the work presented in this thesis seeks to augment.

\subsubsection{Workstation with PCle FPGA Card}

Vendors offer FPGA PCle cards for the hardware-acceleration market [8] [9]. Customers ranging from scientists and researchers to stock traders who wish to accelerate their workload via FPGA can purchase these FPGA boards and plug them into their workstations. These boards tend to contain higher-end FPGA devices, such as the Stratix and Arria families from Intel or Virtex and Kintex from Xilinx. Once plugged into the motherboard of their workstation, communication between CPU and FPGA is facilitated through the PCle lane(s). A PCle driver is installed on the OS to transact PCle packets to and from the FPGA board.

\subsection{CPU-FPGA Hybrid Applications}

Software applications are ones that run on a traditional computer as sequences of instructions that are executed serially by the CPU. FPGA applications are ones that get placed inside an FPGA as a hardware circuit. CPU-FPGA Hybrid applications are ones comprising software and hardware (placed in FPGA) where the two halves work together to accomplish some task(s). 
Hybrid applications allow developers to divide up the workload of their application between the CPU and the FPGA, wherever the strength of each device is beneficial. For example, a developer may run some house-keeping tasks that are run only a few times in the application's execution on the CPU, where the CPU's general-purpose nature allows code to be executed with fairly low overhead. On the other hand, work that is highly repetitive and applied to large sets of data may benefit from running as a specialized accelerator placed in the FPGA, as it may provide better throughput.

An FPGA accelerator typically has the performance advantage over the CPU. The accelerator in the FPGA can be pipelined for the specific task (allowing high pipeline-parallelism) and duplicated multiple times in the FPGA (allowing data parallelism). For example, consider an algorithm that requires 4000 instructions on the CPU. As CPUs are not pipelined to such lengths, the CPU is incapable of fully pipelining the algorithm. If $\mathrm{CPU}$ ran at $4 \mathrm{GHz}$, the $\mathrm{CPU}$ would complete $4 \mathrm{GHz}$ / $4000=1$ Million results per second. On the other hand, if the algorithm was turned into a pipelined circuit on the FPGA capable of yielding a result per cycle, and if the circuit ran at $100 \mathrm{MHz}$, it could yield 100 million results per second. Assuming no data dependency, this circuit could then be duplicated multiple times, allowing even greater throughput.

\subsection{High-Level Synthesis}

When creating a hybrid application, developers can create all parts of the hybrid application from scratch, which has the advantage of being optimizable at the finest granularity, but the disadvantage of being difficult and time-consuming. The most time-consuming part of creating 
the application from scratch is writing the HDL (Hardware Description Language) code to describe the FPGA circuitry. Here, the developer would have to specify their accelerator circuit by placing registers, connecting wires, creating state machines, and so on, which is significantly more time consuming than writing the equivalent functions in a software language.

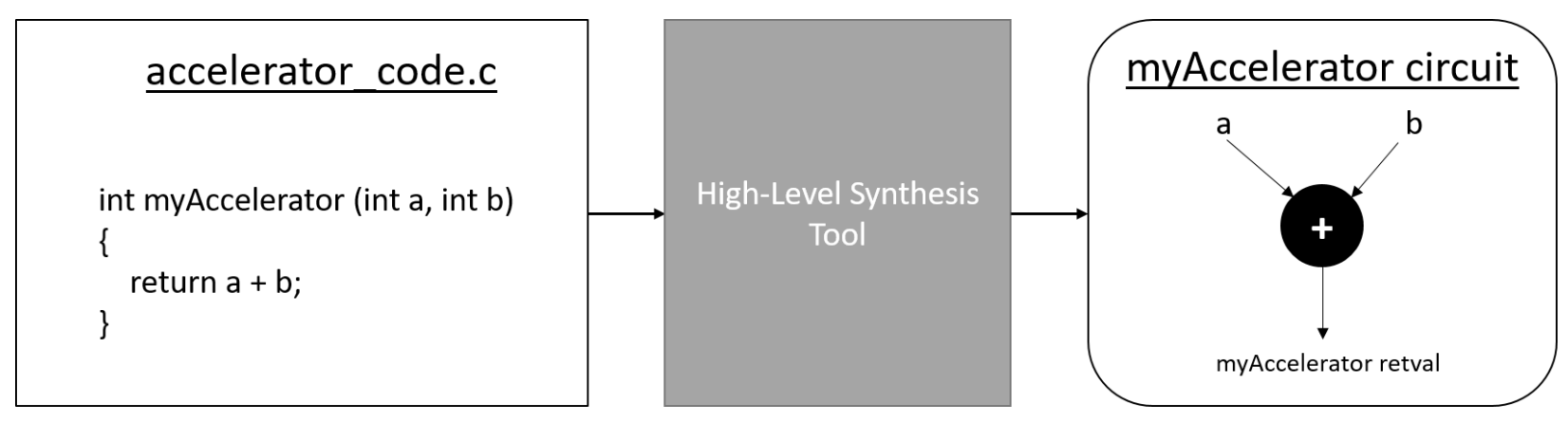

Figure 1 Example of high level synthesis with C-code as input

Instead of writing HDL, developers can use high-level synthesis (HLS) frameworks, such as the Intel OpenCL SDK [4] or LegUp [6]. With HLS, the application is typically written in some highlevel (typically software-language) code to describe the desired logic to be accelerated in the FPGA. The HLS tool then automatically synthesizes the code into a hardware circuit in the FPGA. An example of such synthesis is shown in Figure 1 where C-code describing an integer adder is synthesized by an HLS tool. System-specific details like CPU to FPGA communication, device drivers, and FPGA periphery circuitry are also usually handled by the HLS tool, further reducing development times. HLS tools have been gaining popularity as the performance of the autogenerated accelerators have been deemed "good enough" whilst saving tremendous development time. Furthermore, HLS tools open up the possibility of using FPGAs to software developers who are unfamiliar with HDL. For these developers, they have no option but to use an HLS tool. 
LegUp is an open source HLS tool produced by the LegUp group at the University of Toronto [6].

The tool accepts ANSI C code as the input, converts the code to LLVM IR, then uses LLVM passes to turn the input into HDL. The HDL is then sent through the Intel Quartus tool where it is compiled into an FPGA programming file that contains the accelerators.

Intel OpenCL SDK is a commercial HLS tool sold by Intel Corporation [4]. The tool, as its name suggests, accepts OpenCL code as input, and generates an FPGA programming file containing the accelerator.

\subsubsection{HLS Limitations on System Calls}

Today's HLS tools often place limitations on what can and cannot be synthesized. Common among these limitations is the inability to synthesize system calls. For example, whenever the LegUp HLS tool encounters a system call in the C code to be accelerated, it instantiates an empty HDL module in place of the system call function. One exception in LegUp is printf, which it handles specially by placing a Verilog \$display statement in place. This however does not truly produce a circuit to handle printf. It is instead more of a convenience feature for helping developers debug their resulting synthesized circuits using ModelSim.

The system-call limitation can be a problem, since the input to HLS is usually some software code, and software code tends to contain many system calls (often implicitly) to carry out common tasks like printing to a terminal, and accessing files and devices. To this end, the ability to make system calls from the FPGA is attractive as it could ultimately allow system call-laden software code to be accelerated by high-level synthesis tools. The work presented in this thesis 
works towards achieving an HLS tool that can transparently and seamlessly compile system calls to yield an FPGA accelerator that can make system calls as if it were running on the CPU.

\subsection{Linux Operating System}

Linux is a free, open-source operating system that is found in many kinds of devices such as phones, computers, servers, embedded devices, and cars. There are many distributions of Linux, with some of the most popular being Android, Ubuntu, and Red Hat.

At the core of every Linux distribution is the Linux kernel, which is the program that handles essential OS functionality like scheduling, virtualization, resource allocation, and device drivers. This functionality gives tremendous benefit to developers creating applications. The scheduling and resource allocation means that a developer can run multiple applications on the system and let the OS handle the job of divvying up the resources between them. Device drivers built into the Linux kernel provide support for many widely-used devices, and support for new devices are actively added to the kernel. This means that the developer does not need to worry about low-level interaction with devices. Rather, they can use portable higher-level API provided by Linux to access the devices that are underneath. This further reduces development time of applications.

As the kernel controls the system's resources, one of its jobs is to provide these resources to user-level programs. User-level programs are the ones written by developers that run on top of the operating system. A key difference between a user-level program and the OS is the privilege with which they run. The OS is very privileged - it can access secure (privileged) CPU states, directly access the system's devices, and access any part of memory. For security reasons, the 
user-level program runs without such privileges. Whenever the user-level program needs a privileged service or resource, it cannot access them directly, but instead must request them from the Linux kernel by making a Linux system call.

\subsection{Linux System Calls}

The Linux Programmer's Manual describes the system call as the fundamental interface between an application and the Linux kernel [10]. In practice, a system call is a function that is callable by user processes for requesting some service or resource from the OS. Linux provides hundreds of different system calls, each of which provides a particular resource. For example, the open() system call opens a file in the Linux-managed filesystem, and the write() system call writes data to the opened file. To identify the system call, each one is assigned a unique numerical ID.

When a program makes a system call, it must use the Application Binary Interface (ABI) provided by Linux. The $A B I$ requires a program to follow a calling convention that dictates rules like the number of arguments to the call, to which CPU register each argument is stored, and which CPU instruction is used to initiate the system call. The convention depends on the CPU architecture that is being used, but in general the user program stores the arguments to the system call in the appropriate CPU registers, then calls the architecture-specific system call instruction whilst providing the system call ID. When the system call instruction runs, the CPU changes from user mode to privileged mode, and the ID is used to jump to the appropriate subroutine that handles the system call being requested. 
The goal of this work is to provide an interface that FPGA accelerators can use to request system calls from the Linux kernel, similar to what an $A B I$ provides for software programs. The framework for providing such an interface is described in Section 4. 


\section{FPGA-Initiated Linux System Calls: Potential Use Cases}

\subsection{Filesystem Access}

Often applications access the filesystem to store and read data. When a hybrid application needs to access the filesystem, the common practice today is to do the file accesses in the host program running on the processor. This is illustrated in Figure 2 as the "Traditional Filesystem Access from FPGA". If a read is required, the host program would read a file from the filesystem using the read() system call, and provide the data buffer to the FPGA accelerator. If a write is required, the host program would accept a data buffer from the FPGA accelerator, and write the data to the filesystem using the write() system call.

With the FPGA-initiated Linux system calls, the intermediary host program is no longer required. As shown in Figure 2 as the "Direct Filesystem Access from FPGA", the accelerator would bypass the host program and directly access the file system as needed. This eliminates the accelerator-to-host communication, which potentially reduces latency.

Traditional Filesystem Access from FPGA

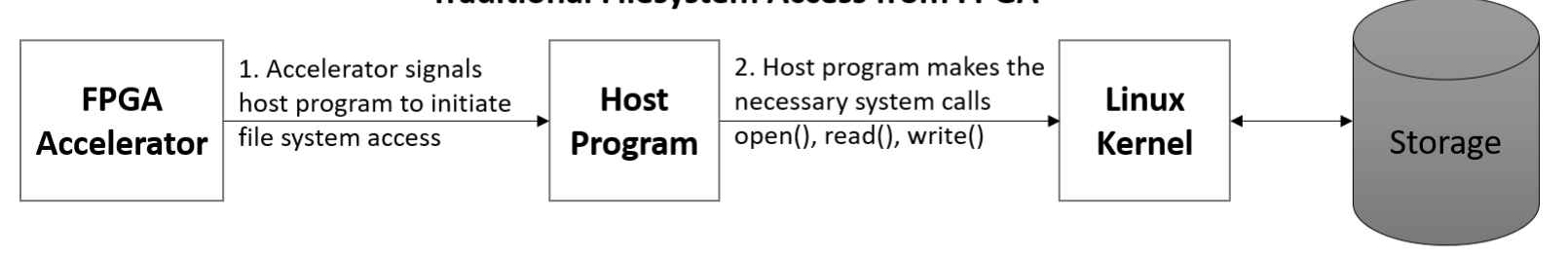

Direct Filesystem Access from FPGA

\begin{tabular}{|c|l|l|}
\hline \multirow{2}{*}{$\begin{array}{c}\text { FPGA } \\
\text { Accelerator }\end{array}$} & $\begin{array}{l}\text { 1. Accelerator makes the } \\
\text { necessary system calls } \\
\text { open(), read(), write() }\end{array}$ \\
\cline { 2 - 2 } & Linux \\
Kernel
\end{tabular}

Figure 2 Filesystem accesses from the FPGA accelerator 


\subsection{Memory Allocation}

In situations where the Linux OS controls the system's main memory, the FPGA accelerator may need to request some memory be allocated for its use. In a user-level program, memory is requested using the malloc() system call. The malloc() calls would return a virtual address (in the calling user process's address space) to the allocated memory buffer which the user program can simply use without any additional work. From the FPGA however, things are not so simple. The malloc() call provides a virtual address in the process's virtual address space. The circuit does not have such a space, as (at least in the case of the Cyclone V SoC used for experimentation in this thesis) the FPGA does not access the memory through the MMU that the Linux kernel does. In other words, the FPGA accelerator accesses main memory via physical addresses, not virtual addresses.

Furthermore, the issue of virtual addresses and physical addresses is not the only problem when trying to allocate memory for an FPGA. When Linux allocates memory, it does so in chunks called pages, which are typically $4 \mathrm{kB}$ long. When Linux allocates memory for a program, it decides mappings between pages in the program's virtual address space to the physical memory. Linux does not guarantee that what is contiguous in virtual memory is also contiguous in physical memory. This is acceptable for a user-level program because whenever it accesses memory through the $\mathrm{MMU}$, it consults page tables to determine which physical address maps the virtual address being accessed. The FPGA, again, does not access the memory through the MMU. This means that if it is granted a memory buffer by the Linux kernel which is longer than a page, the buffer must be contiguous in memory for the FPGA to use it. 
Luckily, the problems outlined above can be solved by using a series of alternate system calls.

The Linux kernel is capable of allocating physically contiguous memory via its Contiguous Memory Allocator (CMA) feature. By leveraging this feature through system calls, an FPGA accelerator could gain a physically contiguous buffer for its purposes. This means that the system memory can be managed by the OS, and the FPGA can gain the memory that it needs by requesting it from the OS just as if it were a software program.

\subsection{Device Access}

Depending on the system topology, various devices may be connected to the CPU-side of the device rather than the FPGA. For example, in the Cyclone V SoC, the CPU-side of the system contains the USB, Ethernet, SDRAM controller, and SDMMC among others. If the FPGA accelerator needed to access one of these devices, it would have to gain access by requesting it from the Linux OS through system calls.

In the case of the Cyclone $\mathrm{V}$ SoC, the hardware manufacturer provides a hardware interface for FPGA-side components to directly control some of these CPU-side devices. However, even if the accelerator possesses the ability to access the devices directly by addressing their register interfaces, it is inadvisable to do this as the Linux kernel's drivers for these devices may contend for them. If the driver and the FPGA accelerator tried to access some device simultaneously in a destructive manner, erroneous behavior is likely to occur. To facilitate the sharing of these devices between accelerators and user-level programs running on the CPU, there must be one broker for these devices, which should be the Linux kernel. FPGA accelerators and user-level programs all must make the appropriate system calls to the kernel for these devices. Various 
system calls may be used for this purpose, including but not limited to ioctl(), read(), and write().

\subsection{Thread-Accelerator Synchronization}

An accelerator and a thread running on the CPU may in some situations need to synchronize. In high-performance compute applications where developers create highly parallelized applications, synchronization between CPU threads and FPGA accelerators could be necessary. When synchronizing between software threads in a multithreaded application, developers typically use library function calls (such as those of the pthread library) to implement the necessary synchronization though mutual exclusion locks, semaphores, and etc. These functions underneath make system calls to guide the Linux kernel to efficiently schedule the threads in the necessary order. System calls used may include timer-related calls like sleep(). By allowing system calls from the FPGA accelerator, a developer using HLS to create the accelerator could potentially use the same synchronization libraries as they would for software to implement thread synchronization in their accelerator. The HLS tool would then synthesize the necessary FPGA circuit to initiate the system calls to the operating system to implement the thread synchronization. 


\section{The FPGA-Initiated Software-Handled Framework}

This section describes the FPGA-Initiated Software-Handled framework for allowing FPGAs to make system calls in CPU-FPGA systems that use the Linux operating system. At a high level, the proposed framework consists of three logical components:

1. Linux host program

2. FPGA accelerator

3. FISH Linux kernel module

The host program runs on the Linux operating system, and is responsible for launching the FPGA accelerator through some interface such as PCle or AXI. The FPGA accelerator carries out its computation and eventually reaches a point in its operation where it requires a system call. The FPGA accelerator then requests a system call from the FISH Linux kernel module. The FISH kmodule runs as part of the Linux kernel and carries out the system call on behalf of the accelerator. When the system call is complete, the FISH kmodule signals the accelerator, and computation on the accelerator resumes. The following sections describe this framework in more detail.

\subsection{A Standard System Call Interface for FPGA Accelerators}

Parallels can be drawn between our framework and the traditional ABI used by software, as shown in Figure 3. The accelerator is analogous to the user program, in that both entities require a resource from the OS. Both the accelerator and the user program must prepare for a system call by providing data in the form of a system call ID and arguments to that system call. 
While the data remains the same, the medium for transfer is different. While the user program directly writes the data to the CPU registers, the accelerator uses some global memory accessible by the CPU. Once the data is ready, the accelerator defers control to the FISH kmodule, similar to how the user program defers control to kernel-mode execution of the system call handler placed in memory by the Linux kernel. Then, the FISH kernel module will carry out the required tasks for providing the accelerator with the requested resources, as the system call handler would normally.

\section{Traditional System Call Convention}



FPGA-Initiated, SW-Handled Convention



Figure 3: Parallels between the traditional system call convention and the FPGA-initiated, SWhandled convention

The end goal is to provide some standardized convention for accelerators to make system calls to the Linux kernel. The precise details of the interface may change over time, especially due to changes in CPU-FPGA system architectures as newer devices are released. If a standard 
interface is achieved, HLS tool vendors and HDL developers can create FPGA accelerators that adhere to this standard making them portable to any CPU-FPGA system running Linux. This is no different than how a Linux binary of a given CPU architecture can make system calls regardless of system specifics like whether an Intel or AMD CPU is present, or which graphics card or hard drive, etc.

\subsection{Handling an FPGA-Initiated System Call}

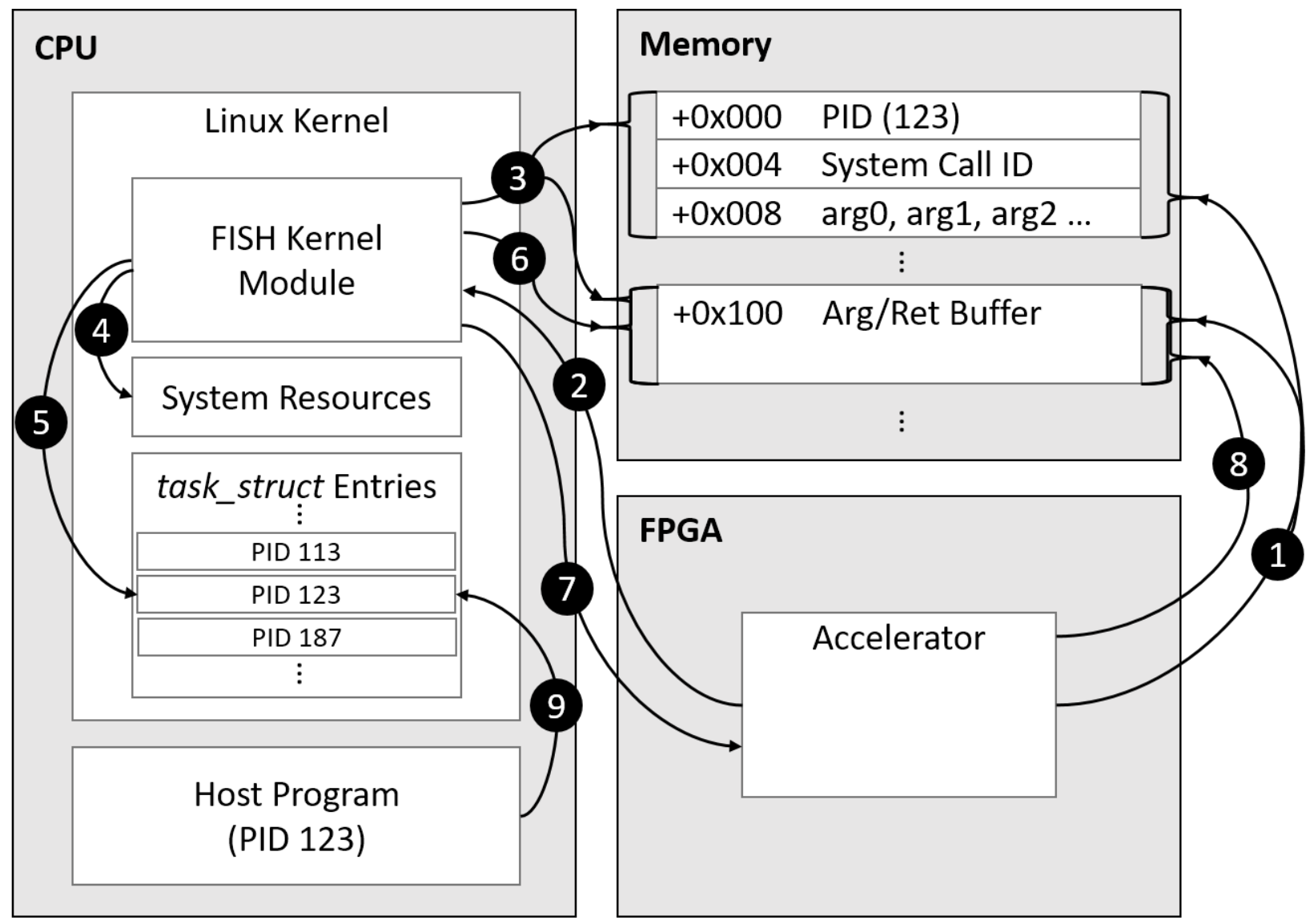

Figure 4: Steps for handling an FPGA-initiated system call

When the FPGA accelerator initiates a system call, it is handled by the process shown in Figure 4. The steps in the process are described below: 
1. Accelerator stores in memory the information needed by the FISH kmodule to handle the system call (host program's PID, system call ID, arguments to the system call, and buffers)

2. Accelerator signals the FISH kmodule to start the system call

3. FISH kmodule reads the information in memory

4. FISH kmodule consumes system resources on behalf of the accelerator

5. FISH kmodule modifies the host program's task_struct object to ensure persistent change as a result of the system call

6. The result of the system call (if applicable) is written to the buffer in memory

7. FISH kmodule signals the FPGA accelerator that the system call has finished

8. Accelerator reads the results of the system call from the buffer in memory (if applicable)

9. The persistent effects of the system call are visible to the host program in its execution further down the line. No special handling is required; system calls naturally access process state stored in the task_struct object.

\subsection{FPGA to Kernel Module Communication}

The framework requires a signaling method between the FPGA and the FISH kernel module for two purposes:

1. To signal the start of a system call (step 2)

2. To signal the completion of the system call (step 7)

The FISH kmodule remains asleep until it receives a signal from the FPGA. Once signaled, it wakes up and carries out the work necessary to handle the system call. When finished, it sends 
the "finished" signal to the FPGA accelerator and goes back to sleep. The accelerator then continues with its computation.

In addition to sending "start" and "finished" signals, the following data must also be passed between the FPGA and the kernel (step 3):

1. Host program's PID

2. System call ID

3. Arguments to the system call

4. Data buffers

The host program's PID must be sent to the FISH kmodule so that it is aware of which host program is associated with the accelerator. An important takeaway here is that although both the host program and the FISH kmodule run in the software domain, they remain uncoupled. Since the FISH kmodule is unaware of the host program, the PID must be provided by the host program to the accelerator and in turn, by the accelerator to the FISH kmodule. Although this seems roundabout, this approach allows a single FISH kmodule instance to provide system call services to multiple hybrid applications running on the system. In other words, a single FISH interface can serve all of the FPGA accelerators, similar to how one ABI can be shared by all user programs.

In addition to the PID, the system call ID is necessary to specify which of the approximately 300 Linux system calls is being requested. Some of these system calls require input arguments, which have to be passed from the accelerator to the FISH kmodule. As some of the arguments 
may be pointers to buffers, memory for these buffers must be allocated and initialized by the accelerator.

In the diagram shown in Figure 4, the data is transferred through system memory. This is a natural way to pass information, as CPU-FPGA systems generally have a global memory which is accessible by both CPU and FPGA. With other system topologies, an alternative communication method may be used.

\subsection{Handling the System Call Request in the FISH Kernel Module}

When a Linux user-level program makes a system call, it switches from executing in user mode to executing in kernel mode through a CPU instruction that requests privileged execution. For example, in the ARM instruction set the SVC (Supervisor Call) instruction is used to change the CPU to the privileged mode. After the switch, the now-privileged process executes the subroutines that carry out the system call. Because the FPGA accelerator does not run on the CPU at all, a completely different approach is required. In our framework, the FISH kmodule is used as the intermediary between system resources/services and the FPGA accelerator. This kmodule runs on the CPU in privileged mode, in place of the accelerator, and executes the necessary subroutines.

The FISH kernel module remains asleep while the FPGA accelerator does not require a system call. When the kmodule is awoken by the accelerator (step 2), it determines which system call is being invoked (via system call ID), and the process for which this system call is being invoked (via PID). The kernel module then invokes modified versions of the system call handler subroutines that correspond to the requested system call. As these system-call subroutines 
were originally written to be called by a user process, they must first be modified before the FISH kernel module can call them. An example of a necessary modification is when a contextspecific call is made by the subroutine (such as one requesting data or settings specific to the current process), which would have different effects being called from a kernel module context rather than from a user process running in kernel mode.

\subsection{Handling System Calls with Persistent Effects}

Some system calls have a persistent effect on the calling process. For example, a call to open() would open a file and allocate a file descriptor to that file. The file descriptor then remains reserved for the duration of the process's execution, or until close() is called on that file descriptor. Clearly, some persistence is required when handling system calls. Further, for the FPGA-initiated call to behave seamlessly (indistinguishable from a SW-initiated system call), the persistent change must be made visible not only to the FPGA accelerator, but also to the host program (step 9). Simply put, a file opened by the FPGA accelerator must be accessible by the software, and vice versa.

To keep track of the running processes and their various state data, the Linux kernel uses a table of task_struct objects. In this table is a task_struct object for every process, which keeps track of process-specific information like execution state, file descriptor tables, child processes, etc. To achieve the required persistent effects of system calls, the FISH kmodule modifies the host process's task_struct object (step 5). Then, any system call further down the line (whether it is software or FPGA initiated) consults the task_struct to see the persisting effects of prior system calls. 
A special case that has to be handled with regards to the task_struct table is when the accelerator is designed to be standalone (run without a host program). In such a case, we propose that a placeholder process be created for that accelerator to hold necessary states. If an accelerator makes a system call without its PID register being initialized (IE having a value of 0) then the FISH kernel module can detect this and spawn the necessary placeholder process. Then the FISH kernel module fills in the PID register with the PID of the placeholder process that it has just spawned, linking the placeholder process to future system calls made by the standalone accelerator. 


\section{Framework implementation}

This section describes the completed proof-of-concept implementation of the proposed framework running on an Intel DE1-SoC board containing the Cyclone V SoC (which includes an ARM Cortex-A9 CPU) and 1GB of DDR3 memory. A custom Linux distribution with kernel version 3.18 was run on the ARM processor and DDR3 memory. The DDR3 memory was also used as the global memory, to transfer data between the CPU and the FPGA. The Cyclone V SoC contains an ARM Generic Interrupt Controller (GIC) that allows FPGA to CPU interrupts. These interrupts were used for the FPGA to FISH kmodule signaling.

As a proof-of-concept demonstration, four common system calls for accessing the file system, open(), read(), close(), and write(), were implemented. The ability to access the Linux filesystem from the FPGA has been of interest to researchers in the past [11]. We describe the code needed in the host program code for launching the accelerator, the HDL necessary in the FPGA accelerator to initiate the system calls, and the FISH kernel module code for handling the system calls.

\subsection{Intel DE1-SoC Development Board}

The Intel DE1-SoC board was chosen as the test platform for the FISH framework. This board was chosen as it was supported by LegUp HLS (the tool which we used to synthesize the accelerator $\mathrm{HDL}$ ), and provided the necessary CPU-FPGA coupling required to demonstrate the framework. The board is manufactured by Terasic Technologies [9] and contains an Intel Cyclone V SoC device. The SoC device contains a dual-core Cortex-A9 processor running at 925 
$\mathrm{MHz}$, and FPGA fabric providing $85 \mathrm{k}$ logic elements. The board's features are outlined in Figure

5.

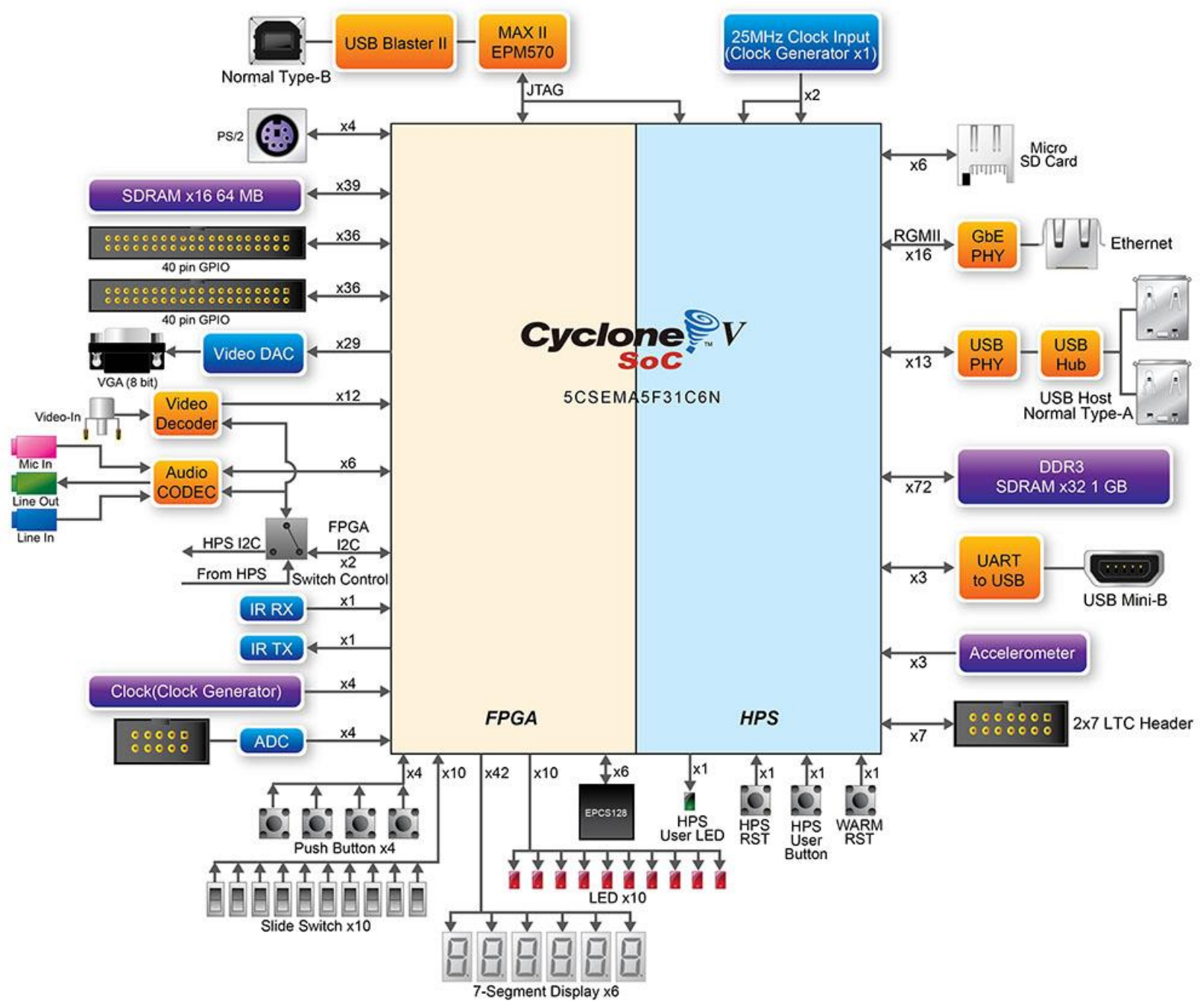

Figure 5 Block diagram of the DE1-SoC board [12]

The features of the board necessary to demonstrate the FISH framework are:

- ARM Cortex-A9 Dual Core processor for running Linux, host program, and the FISH kernel module 
- 1 GB DDR3 SDRAM for running the programs as well as being the buffer for FPGA to CPU data transfers

- MicroSD card slot for booting Linux from the SD card

- Gigabit Ethernet for developing code on the board via SSH and FTP

- UART to USB for serial terminal communication

\subsection{The DE1-SoC Linux Distribution}

A custom distribution of Linux designed for the Intel DE1-SoC board was created and used for the proof of concept implementation. The distribution is based on Terasic Technologies' Ubuntu Linux image, but contains an updated Linux kernel version 3.18 instead of the default 3.12. When compiling the Linux kernel, the following key features were enabled (the complete config file used when compiling is provided in Section 9.9):

- Contiguous Memory Allocator (CMA) support, with 256MB reserved for CMA

- Intel/Altera FPGA programming drivers and support

The CMA support is necessary for allocating physically contiguous memory. Our accelerator requires physically contiguous memory as it does not contain a Scatter-Gather DMA (SGDMA). The Intel/Altera FPGA programming support is used to program the FPGA with the accelerator before running the host program. Having this feature meant that all of the requires tasks of running a hybrid application could be done on the board itself without intervention of a host workstation. This allowed creation of scripts that automated the process of running a hybrid application, which included tasks like programming the FPGA, inserting the FISH kernel module, running the CMA allocation driver, and running the host program. 


\subsection{Allocating the Shared Data Buffers}

In order to pass data between the accelerator and the FISH kernel module, the DE1-SoC board's DDR3 memory was used as the shared global memory. Since the Linux operating system runs on and manages this memory, it was necessary to allocate buffers before transferring data. Allocating memory using a normal malloc() was problematic, as the allocated memory could be paged and scattered in memory, and the accelerator cannot read discontiguous pages in memory. To solve this problem, Linux's Contiguous Memory Allocator (CMA) was used to reserve a contiguous stretch of memory.

\subsection{Implementing the Host Program}

The host program launches the accelerator using its memory-mapped register interface, which is access through the HPS-to-FPGA bridge. The memory-mapped interface is accessed by mapping physical addresses to virtual address using /dev/mem and mmap(). Once mapped, its registers are accessed by the host program. The only implementation detail worthy of mention here is the need to call getpid() and pass the program's PID to the accelerator before launching it.

\subsection{Implementing the Accelerator}

The LegUp HLS tool was used as a starting point to generate the accelerator. The C-language function shown in Figure 6 was accelerated using the HLS tool. 




Figure 6: C-language function that was accelerated using LegUp HLS

LegUp creates a memory-mapped register interface for the accelerator that contains control and status registers that allow the host program to launch it and determine its state. The generated accelerator's memory-mapped interface was connected to the Cyclone V SoC's HPSto-FPGA bridge using the Intel Qsys tool [13]. By default, LegUp's register interface contains the 64-bit DATA register for storing the return value of the accelerator, a STATUS register that holds bits for monitoring the accelerator's status, and ARGX registers for passing arguments to the accelerator. In addition to the default registers, the following registers were manually added:

1. PID

2. System Call ID

3. System Call Arguments $0-3$

4. System Call Return Value

This resulted in a final register interface as shown in Figure 7.

\begin{tabular}{|l|l|}
\hline Offset & \\
\hline$+\mathbf{0} \times \mathbf{0 0}$ & DATA \\
\hline $\mathbf{+ 0 \times 0 8}$ & STATUS \\
\hline $\mathbf{+ 0 \times 0 C}$ & SC_ID \\
\hline $\mathbf{+ 0 \times 1 0}$ & SC_STATUS (not used for open, close, write) \\
\hline $\mathbf{+ 0 \times 1 4}$ & SC_RETVAL \\
\hline $\mathbf{+ 0 \times 1 8}$ & SC_PID \\
\hline $\mathbf{+ 0 \times 1 C}$ & SC_ARG0 \\
\hline $\mathbf{+ 0 \times 2 0}$ & SC_ARG1 \\
\hline
\end{tabular}




\begin{tabular}{l|l}
$\mathbf{+ 0 \times 2 4}$ & SC_ARG2 \\
$\mathbf{+ 0 \times 2 8}$ & SC_ARG3
\end{tabular}

Figure 7 Register interface of the FISH-compatible accelerator

The system-call related registers have the prefix SC_. The PID register is written by the host program, and is later read by the FISH kernel module to determine the host program's PID. The system call ID register is written by the accelerator, and contains the unique numerical ID of the system call being requested. This register may be written multiple times, as a single accelerator may request multiple different system calls during its execution. The argument registers hold the arguments to the system call. Any pointer arguments in these registers hold physical addresses (in the CMA-allocated memory). Note that in a full-fledged implementation, there may have to be as many as seven argument registers (for a small number of Linux system calls that accept seven arguments), but only four were added in our implementation as they were sufficient for open(), read(), close(), and write(). The return value register is used by the FISH kernel module to pass on the return value of the completed system call to the accelerator. A write to this register doubles as the finished signal, telling the accelerator that the system call has completed.

The LegUp tool instantiates a Verilog module for each C-language function that is synthesized. Since LegUp does not support synthesis of system calls, it instantiates an empty Verilog submodule in place of the system call. It then continues to generate the rest of the accelerator circuit, including the logic for controlling the submodule and for consuming the submodule's result. This means that if the empty placeholder submodule is implemented, the rest of the accelerator works as intended. The submodules for open(), read(), close(), and write() were 
implemented by hand. These submodules were made to set the accelerator's registers (mentioned above), initialize argument buffers in the CMA memory if required, and send the interrupt request to the kernel module (via the ARM Generic Interrupt Controller) to trigger the system call. As an example, the steps carried out by the submodule for write() are listed below:

1. Write the system call ID of write(), $0 \times 4$, into the System Call ID register

2. Write the arguments $\mathrm{fd}$, buf, and count into the argument registers

3. Write the buf string into the buffer pointed to by the buf pointer

4. Send the interrupt request to the FISH kernel module

5. Wait until the return value register is written by the FISH kernel module

Due to excessive length, HDL code generated by LegUp is not included in this document. However, the HDL code for the open, write, and close modules are provided in Section 9.6, 9.7, and 9.8 respectively.

\subsection{Implementing the FISH Kernel Module}

The FISH kernel module was implemented to handle the system calls requested by the accelerator. When the kernel module is loaded into the kernel, it registers an IRQ handler using the call to request_threaded_irq as shown in Figure 8. This registers a threaded interrupt handler which executes the function irq_handler. It is critical that the handler is threaded, as a threaded handler is interruptible. This is necessary for calling many of the system call handler functions, which have code that can only be executed from an interruptible context. For example, code that requires thread synchronization functionality (locks, semaphores) can only 
work if the thread itself can be interrupted by the Linux scheduler, allowing the other threads to run.

request_threaded_irq(72, NULL, (irq_handler_t)irq_handler, IRQF_TRIGGER_HIGH | IRQF_ONESHOT , "irqhandler", (void *)(irq_handler));

Figure 8: Registering a threaded IRQ handler

An undesired side effect of using a threaded interrupt handler is that the IRQ is lowered immediately upon waking the handler thread. This is fine for certain interrupts where the required work can be queued and completed further down the line by the threaded handler. Unfortunately, this is not acceptable in our situation due to data dependencies. As computation further down the line may depend on the result of a system call, we must ensure the system call completes before lowering the IRQ. To solve this, the IRQF_ONESHOT flag is used, which forces the IRQ high until the threaded handler finishes.

Abbreviated code for the irq_handler function is shown in Figure 9. It first reads the accelerator's registers. Using the PID, the handler gets the task_struct object corresponding to the host program. By using the System Call ID register, it determines which system call is being requested and calls the appropriate handler function (sys_write_handler, sys_read_handler, sys_open_handler, or sys_close_handler) in the switch case block. Each system call handler function is passed the task_struct object, as well as the arguments. For pointer arguments, the phys_to_virt function is used to convert the physical address (written by the accelerator) to the kernel space virtual address. These virtual addresses can then be directly dereferenced by the handler functions to access the buffers to which they point. 




Figure 9: IRQ handler code (abbreviated)

Abbreviated code for sys_open_handler is shown in Figure 10. The full code for the handler is shown in Section 9.2. The host program's task_struct object is used to get its files table (files_struct). Using a modified version of the Linux function __alloc_fd, a file descriptor is allocated. If the provided filename argument is a full path, it is used without modification. If it is an implicit path, then it is treated as a relative path from the host program's current working directory (CWD). The CWD is retrieved using task_struct's fs member object. Once the path is 
determined, the file is opened and installed into the host program's file descriptors table using

fd_install.

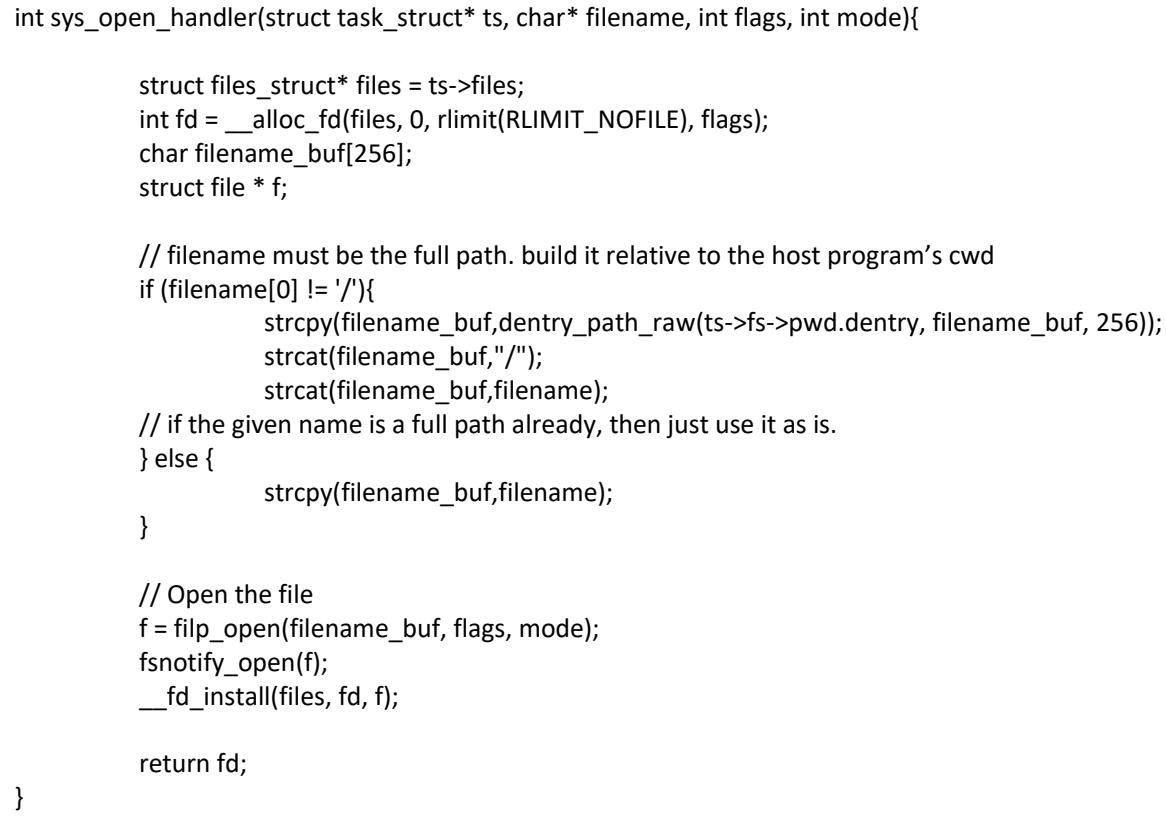

Figure 10: Abbreviated code for the open system call handler

Code for the other handlers are provided in Section 9.2. 


\section{Assessing the Framework Implementation}

\subsection{Verifying Correct Behavior}

The implemented accelerator makes two calls to open() to open files named input.txt and output.txt within the current working directory (CWD) of the host program. It then calls read() to read the contents of input.txt then calls write() to store the data that was read into output.txt. Finally, the program calls close() to close the two.

This program allows us to verify key functionality. First, it tests whether an accelerator can request the allocation of a file descriptor using the call to open(). Second, it tests whether the accelerator shares the same "context" as the host program, which in this case was the CWD of the host program. This was verified by executing the host program from different directories, and seeing that the file was opened in the correct location. This is validation that the technique of referencing the host program's task_struct from the kernel module is effective for achieving a single cross-domain hybrid application state. Third, the read() and write() verify that an accelerator can access the Linux filesystem. Indeed, after running the accelerator, the output file contained the correct text. Finally, the close() verifies that an accelerator can deallocate a file descriptor.

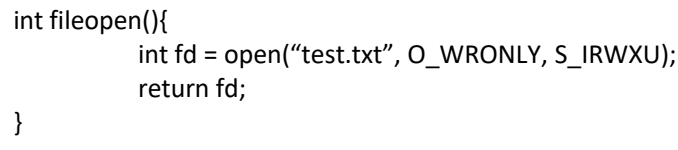

Figure 11: An accelerator that opens a file and returns the file descriptor 
To test cross-domain persistence, a second host program and accelerator (shown in Figure 11) were created. This accelerator was created to open file and return the allocated file descriptor (without writing to the file or closing it). To ensure that the open from the accelerator correctly modified the host program's state in the task_struct and verify that this state was visible in the software domain, the file descriptor returned by the accelerator was used by the host program to then write to the file. This worked correctly, indicating that the accelerator's open() system call correctly opened the file and added it to the host program's file descriptor table in its task_struct object.

\subsection{Performance}

The latencies of the software and accelerator-initiated versions of the system calls open(), $\operatorname{read}()$, close(), and write() were measured. The latencies of the software-only system calls were measured using the ARM processor's timers, accessed with gettimeofday(). These latencies represent the best possible system call runtimes, and the FISH interface aims to achieve latencies as close to them as possible.

The latencies of the accelerator-initiated system calls were measured using the SignalTap signal analyzer to count the number of accelerator clock cycles elapsed between sending the start signal to the system call submodule, and receiving the finished signal. The number of cycles was then divided by the accelerator clock rate $(50 \mathrm{MHz})$ to obtain the "HW-Initiated (Total)" times shown in Figure 12. Similarly, a cycle count was also recorded between sending the interrupt to the FISH kernel module and receiving back a response, to measure how long the kernel module 
took to handle each system call. This time is shown as the "HW-Initiated (Kmodule)" time in Figure 12.

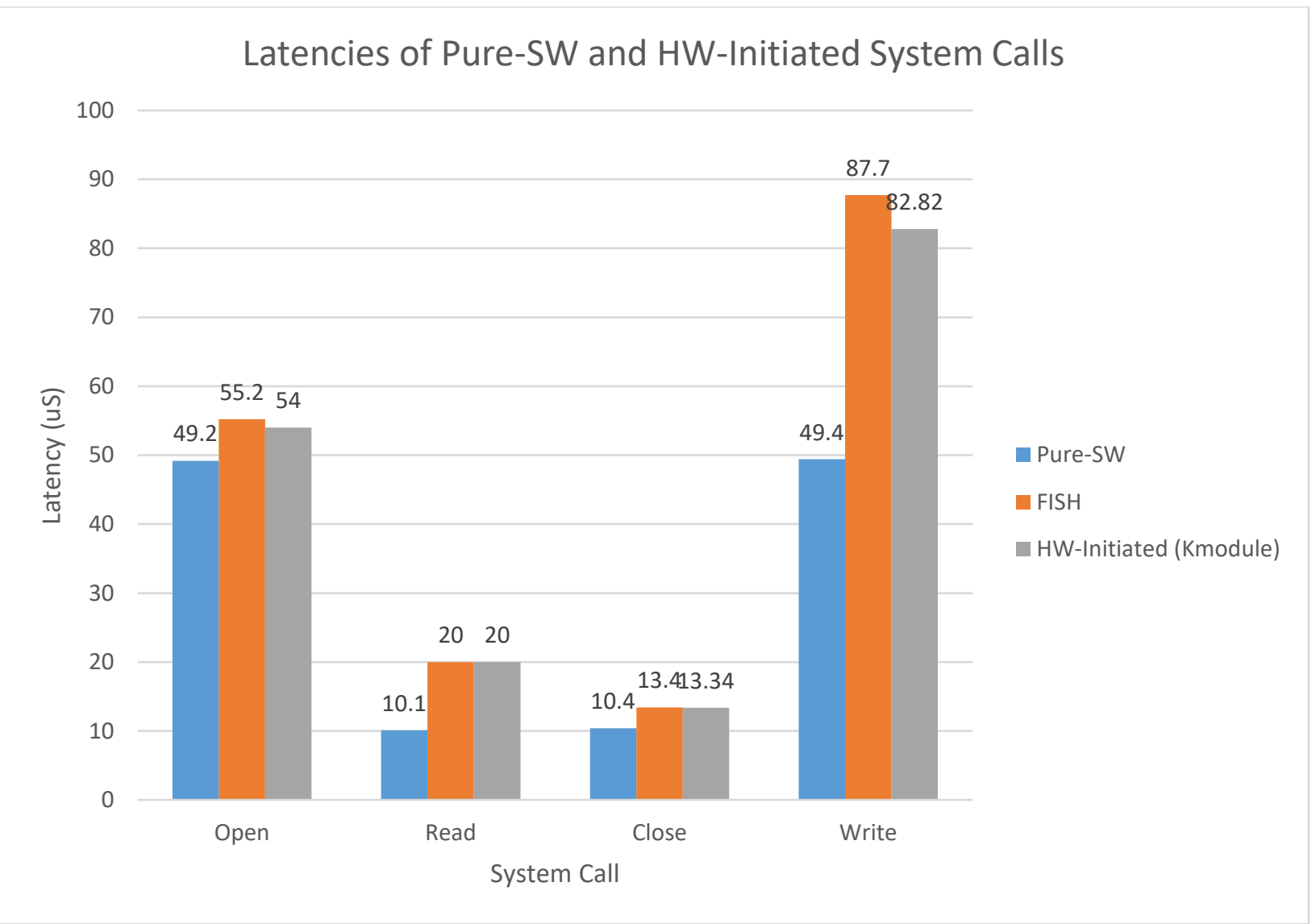

Figure 12: Latencies of Pure-SW and HW-initiated system calls

The measured latencies show that the open(), read(), close(), and write() FPGA-initiated system calls take roughly $1 \mathrm{x}-2 \mathrm{x}$ as long to finish compared to their software-only counterparts. The system calls that took roughly $2 x$ longer than their software-only counterpart had one commonality, which was the need for an intermediate buffer. Intermediate buffers were required here because the Linux implementation of their handlers expected user memory space buffers for the strings, which the FPGA accelerators did not provide. As such, an intermediate 
buffer was allocated by the FISH kernel module to provide the adequate memory-space buffer to the underlying system call handler.

Although the performance numbers measured cover only four system calls out of hundreds that Linux provides, we believe they indicate the neighborhood of performance that is achievable. We consider the numbers to be promising. 


\section{Related Works}

Other works have studied FPGA-to-OS interfaces running on CPU-FPGA systems. For example, ReconOS [14] is a RTOS which provides system resources to hardware in the FPGA. Their approach uses a hardware interface in the FPGA which communicates with an interrupt handler on the CPU side, much like our work. Instead of using the interrupt handler to handle the system call request immediately, it wakes up a user-level delegate thread which is spawned for each hardware thread by ReconOS. The delegate thread then requests the system resource from the OS, on behalf of the FPGA.

HThreads [15] is another RTOS whose goal is to allow tighter interaction between software and hardware threads. A special scheduler in HThreads allows synchronization between software and hardware threads. This approach could allow delegated system call access to the FPGA by running a software thread whose sole job is to make a system call. This thread would block (using the OS's software-to-hardware synchronization capability) until the hardware thread needs the system call.

Thomas et al created the PushPush [16] linker to allow linking of software and hardware components into a single application. Using PushPush, an application of interleaved software and hardware computation can be created, meaning the software portion could make system calls. ReconOS, HThreads, and PushPush offer a system call mechanism that effectively uses a user-level thread to make the call on behalf of the FPGA. This approach is equivalent to having a host program that makes system calls on the accelerator's behalf, rather than allowing the accelerator make the call itself. 
BORPH [17] is a modified Linux operating system that presents hardware interfaces in FPGA which serve as message passers to the Linux kernel. Their work focuses on providing UNIX file system access to FPGA circuits [11]. An important difference is that their interface provides direct FPGA to OS filesystem access, with no interaction between a host program and the accelerator. 


\section{Conclusions and Future Work}

The work presented in this thesis aims to be a starting point for defining a standardized Linux to FPGA system call interface. As such, there are many small details that remains to be solved. The work instead gave ideas on the potential interface at the high level, giving insights on the general-case interactions between FPGA and Linux, and an implementation of a limited number of system calls on one of many currently available CPU-FPGA architectures. To achieve an industry-ready interface with all detailed fleshed out would require far more work. For one, it would probably require some collaboration of hardware vendors (CPU, FPGA, interconnect), Linux developers, and Linux users. Furthermore, there are many CPU-FPGA topologies to be considered with more than just those mentioned in Section 2.1. For example, IBM has a CPUFPGA interface in their POWER8 processors called CAPI [18], and Microsoft has its Catapult system [19]. 


\section{Appendix}

\subsection{Host Program Code}

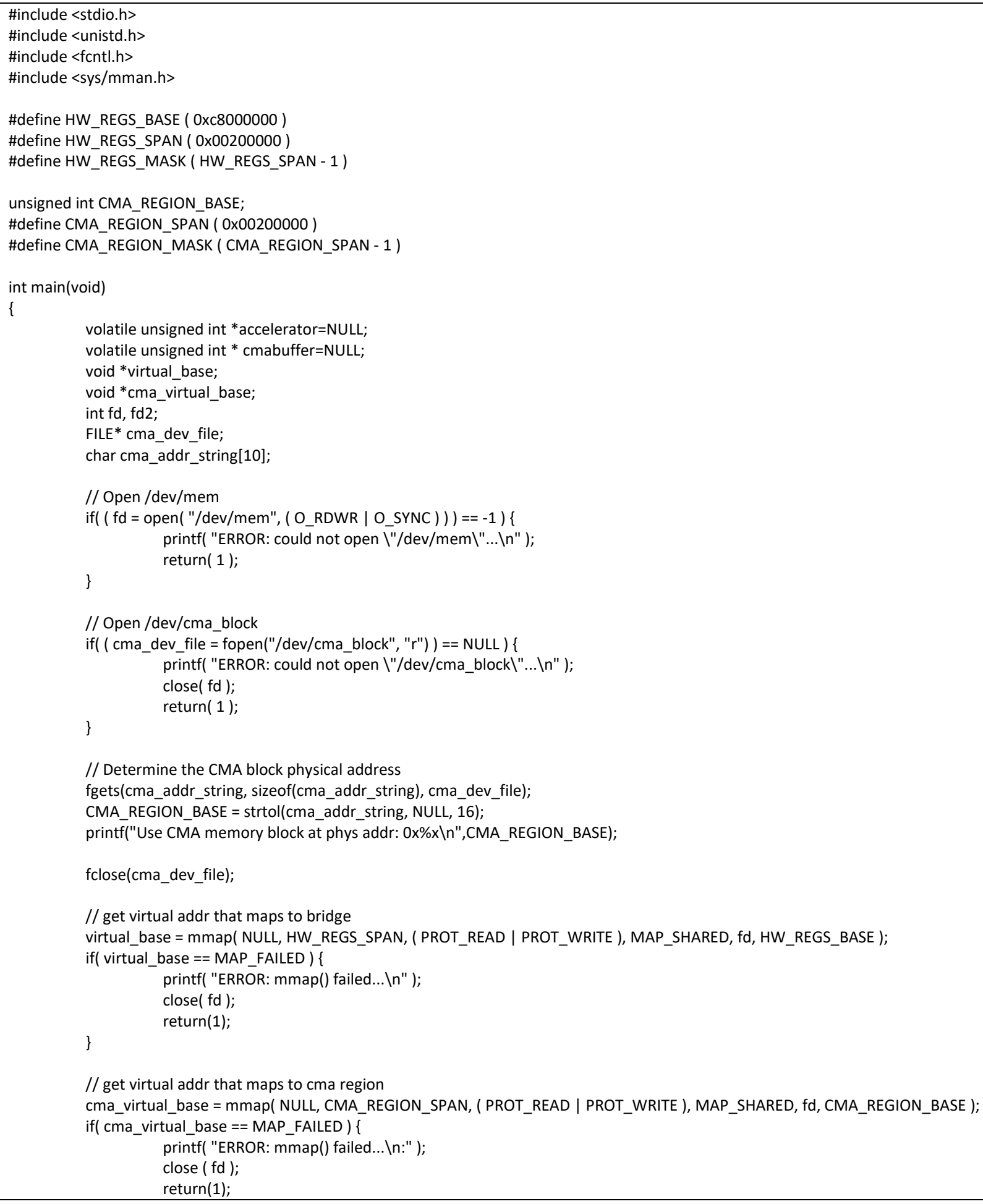




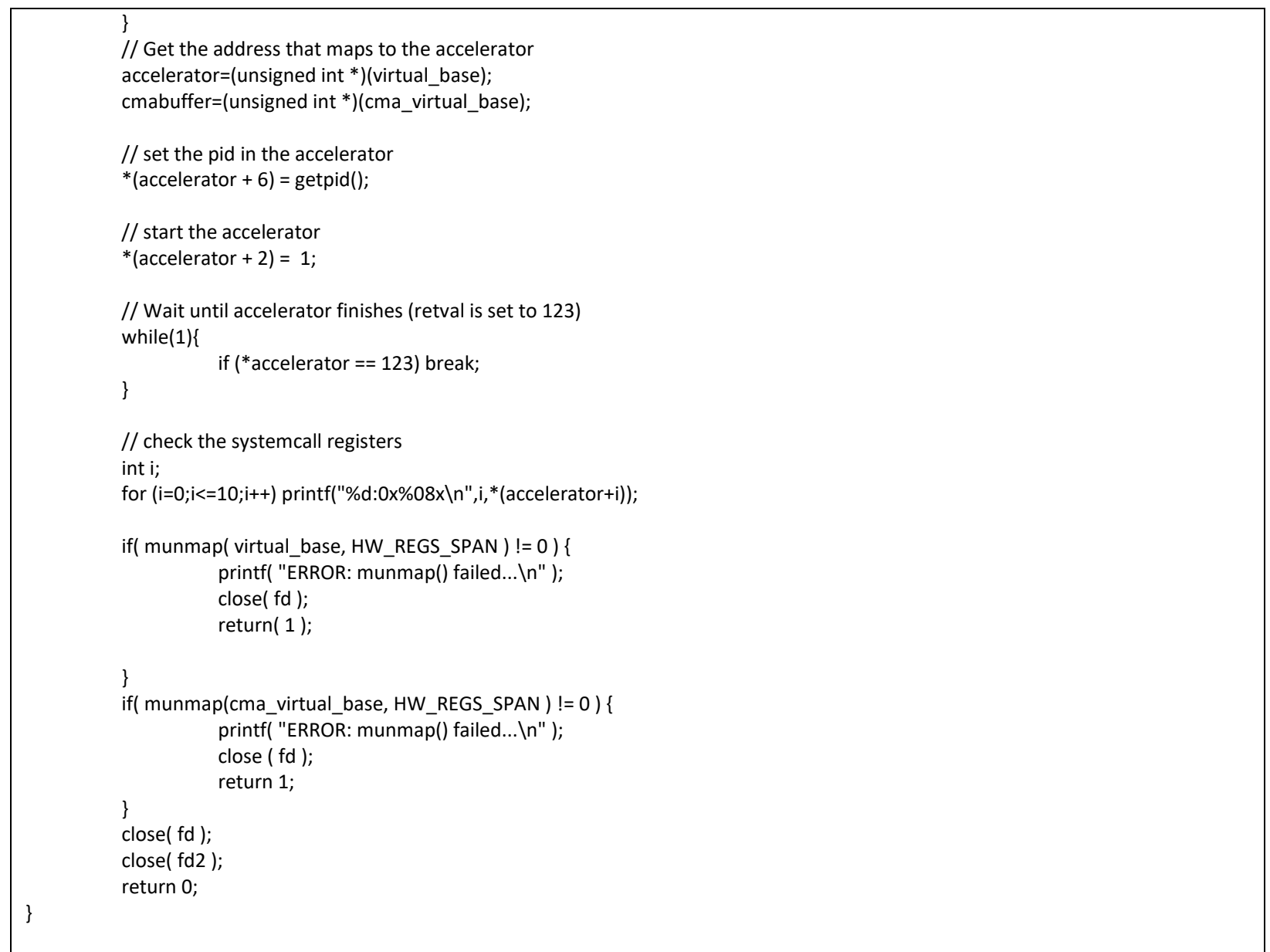

Figure 13 C-language code for the host program that launches the accelerator

\subsection{FISH Kernel Module Code}

\subsubsection{Main Kernel Module Code}

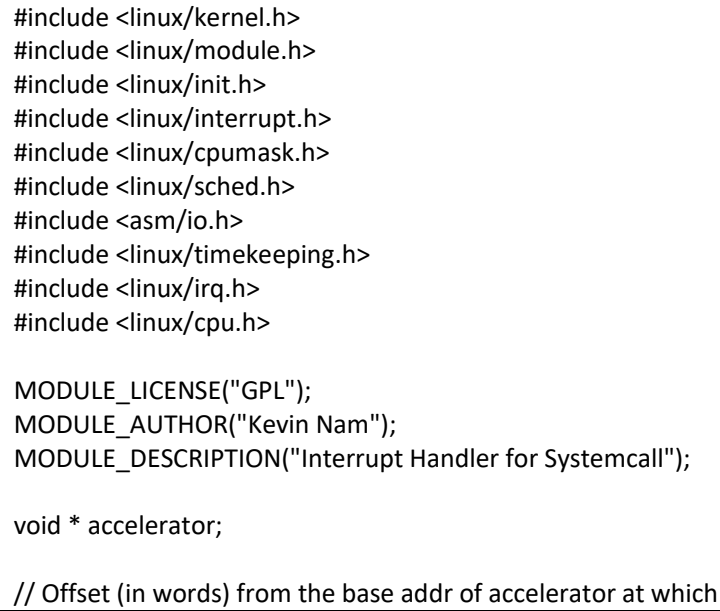




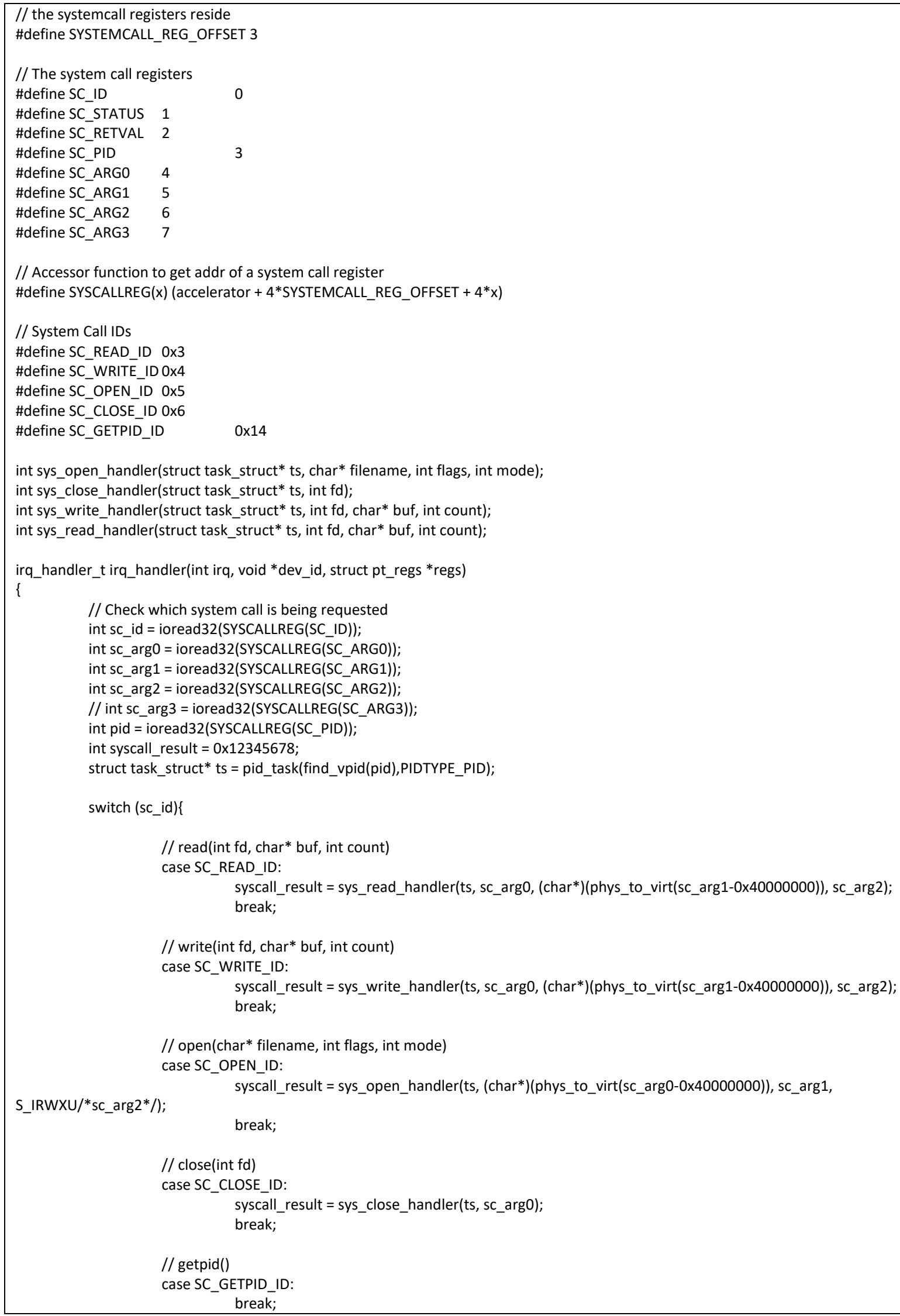






\subsubsection{Open() Handler}

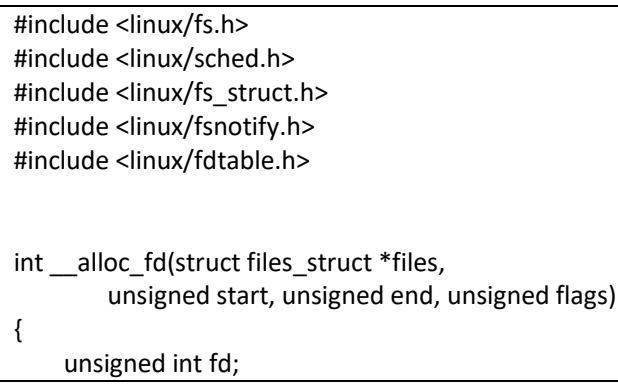











\subsubsection{Read() Handler}

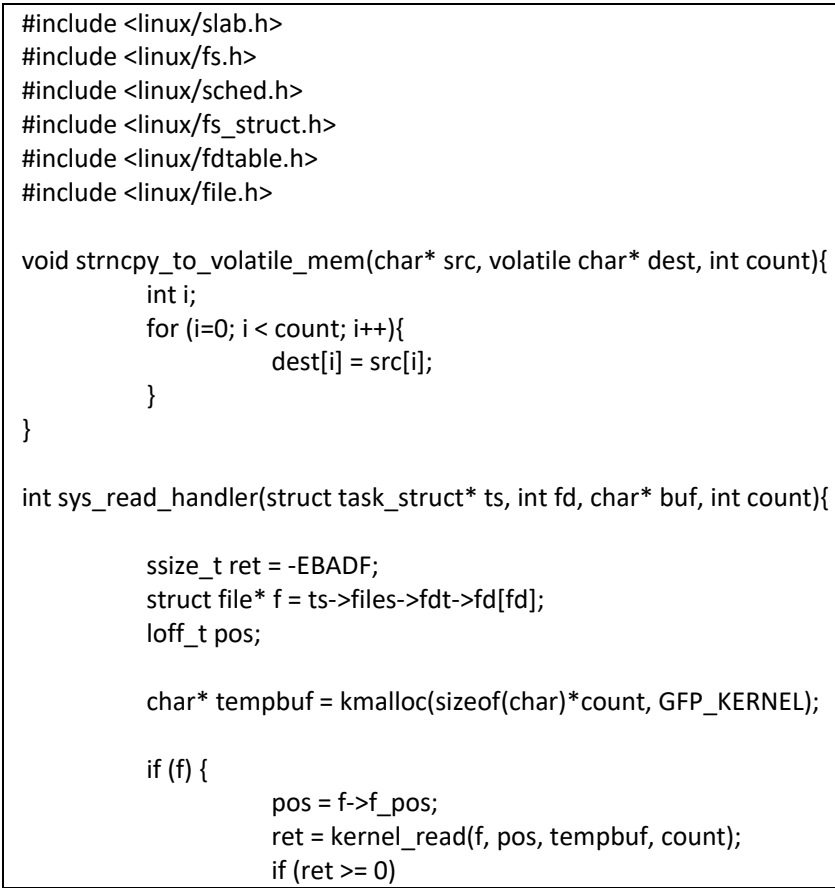




\}else $\{\quad$ f->f_pos = pos;
\}$\quad$ printk(KERN_ERR "ERROR: $f=$ = NULL. NOT WRITING ANYTHING! In");
strncpy_to_volatile_mem(tempbuf, (volatile char*) $)$ buf, count);
kfree(tempbuf);
return ret;

\subsubsection{Write() Handler}



\subsubsection{Close() Handler}

\#include <linux/fs.h>

\#include <linux/sched.h>

\#include $<$ linux/fs_struct.h $>$ 




\subsection{CMA Driver Kernel Module Code}

This kernel module allocates physically contiguous memory for the accelerator to use. The physical address is printed through the character device interface so that the user program (the host program) can determine the physical address of the contiguous buffer. 


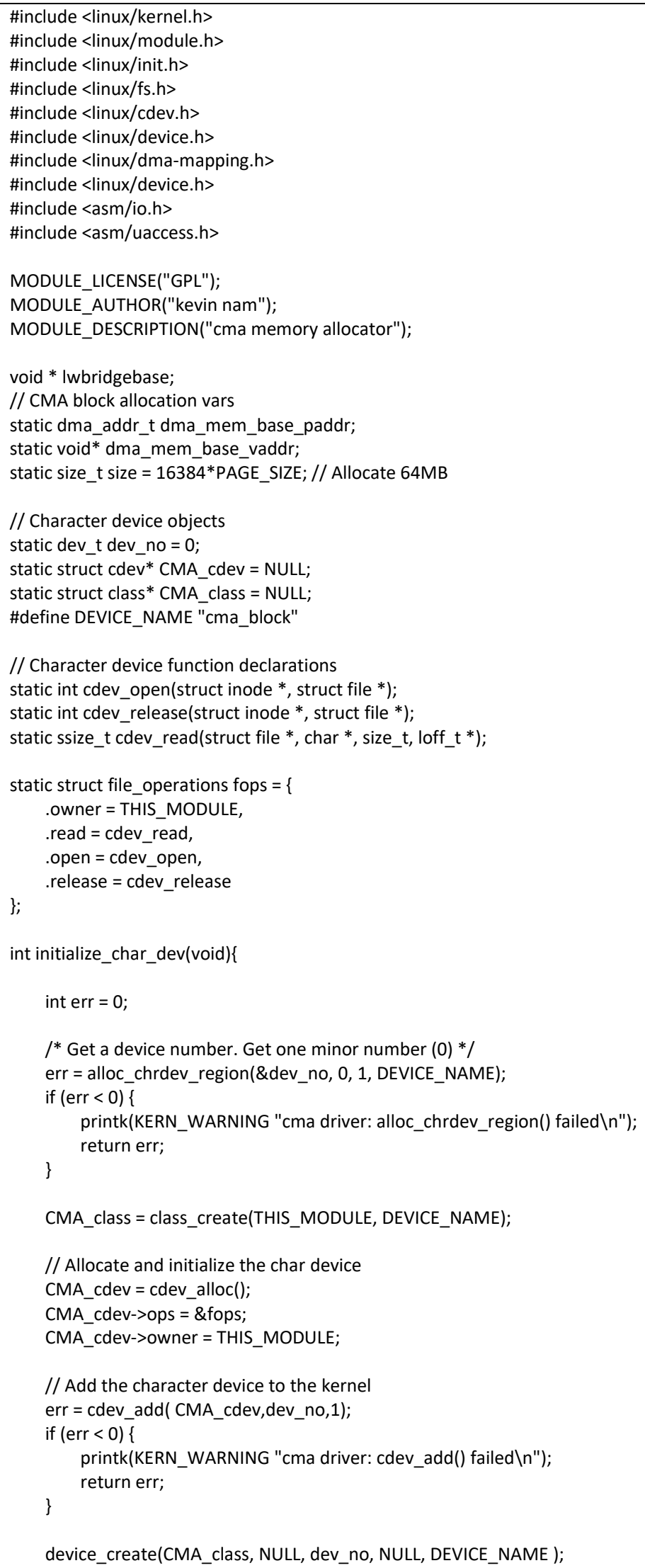




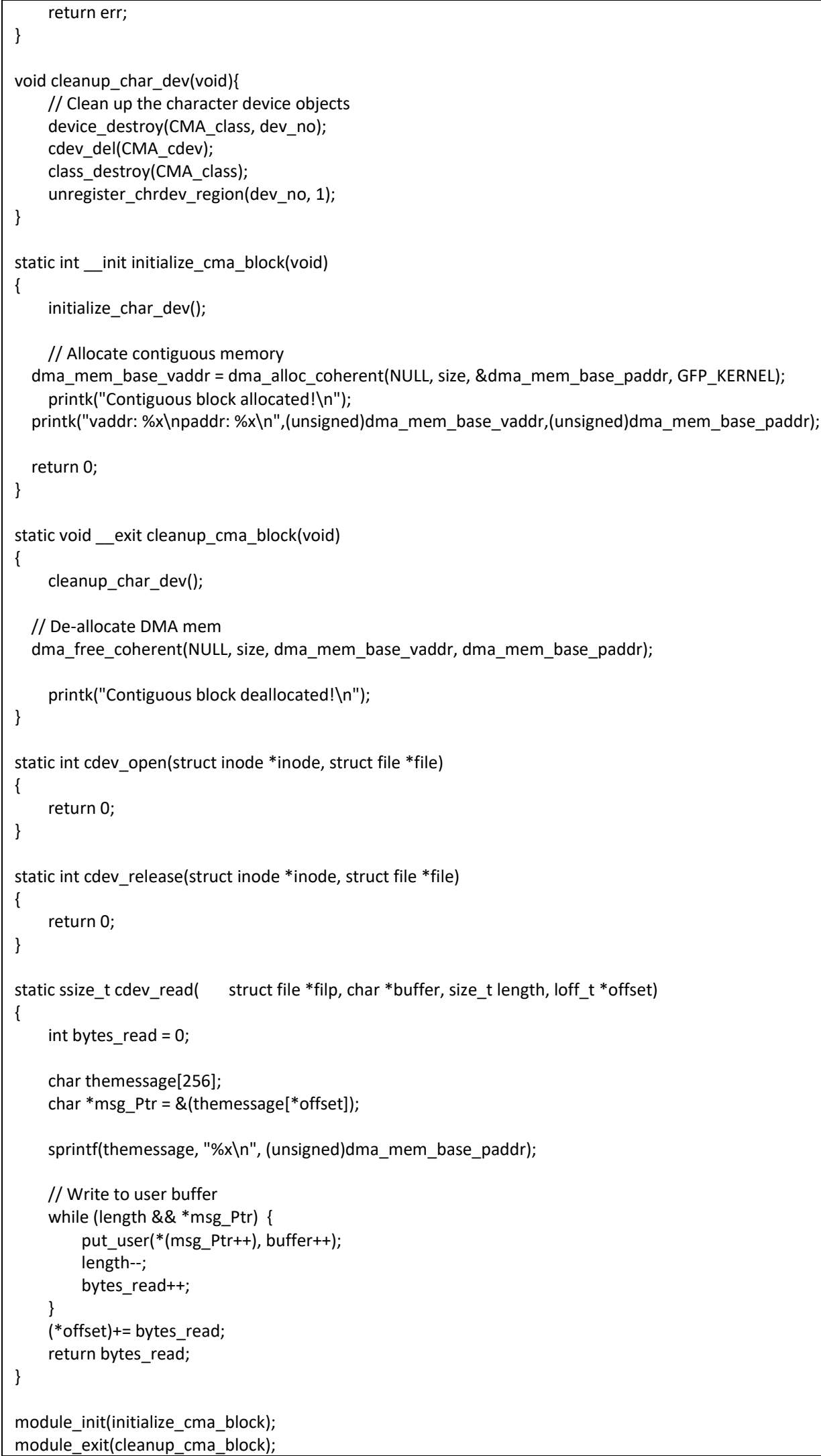

Figure 14 Kernel module code for allocating physically contiguous memory using CMA 


\subsection{LegUp C-Language Code for Accelerator}

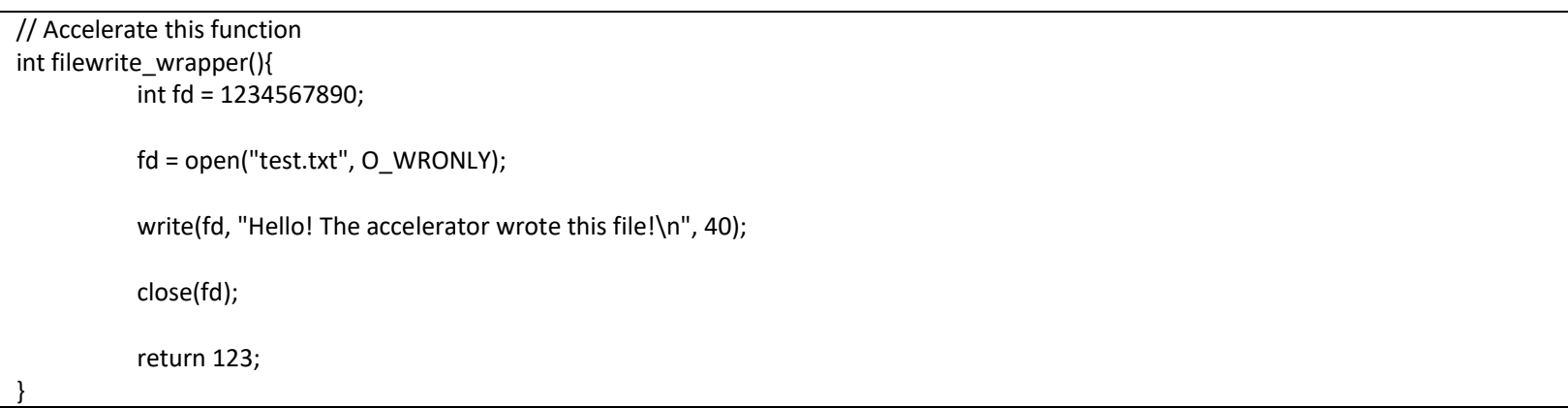

Figure 15 Accelerator code (in C) that was synthesized using LegUP

9.5 LegUp-Synthesized Accelerator Code in Verilog HDL

Omitted due to length. Contact author (kevin.nam at utoronto ca) for the code.

Figure 16 Accelerator code (in Verilog HDL) that was generated by LegUP

\subsection{Verilog HDL Module that Initiates the Open System Call}

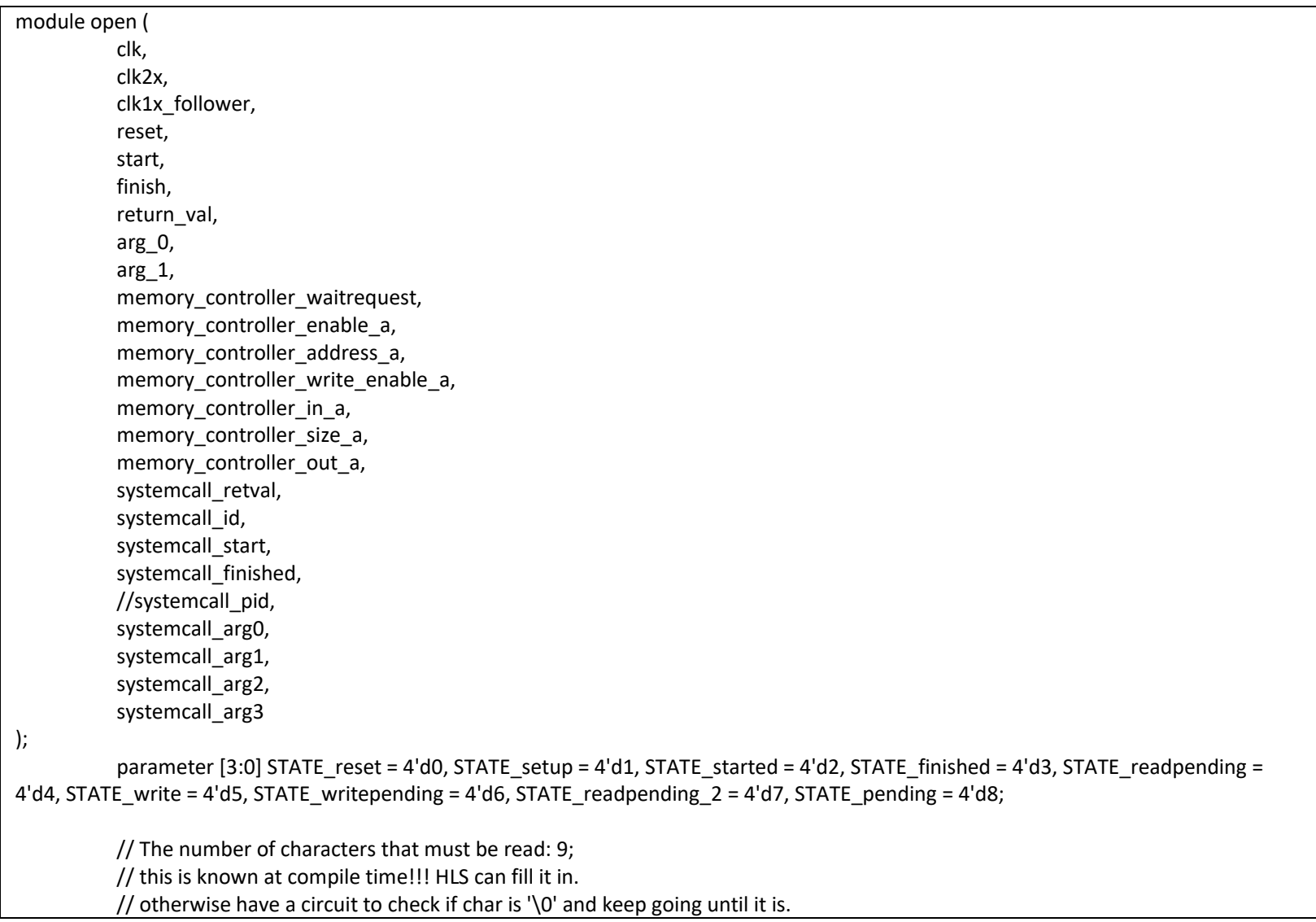


parameter [7:0] length = 8'd9; //

input clk;

input clk2x;

input clk1x_follower;

input reset;

input start;

output reg finish;

output reg [31:0] return_val;

input ['MEMORY_CONTROLLER_ADDR_SIZE-1:0] arg_0;

input ['MEMORY_CONTROLLER_ADDR_SIZE-1:0] arg_1;

input memory_controller_waitrequest;

output reg memory_controller_enable_a;

output reg ['MEMORY_CONTROLLER_ADDR_SIZE-1:0] memory_controller_address_a;

output reg memory_controller_write_enable_a;

output reg ['MEMORY_CONTROLLER_DATA_SIZE-1:0] memory_controller_in_a;

output reg [1:0] memory_controller_size_a;

input ['MEMORY_CONTROLLER_DATA_SIZE-1:0] memory_controller_out_a;

input [31:0] systemcall_retval;

output reg [8:0] systemcall_id;

output reg systemcall_start;

input systemcall_finished;

//output reg [31:0] systemcall_pid;

output [31:0] systemcall_arg0;

output reg [31:0] systemcall_arg1;

output reg [31:0] systemcall_arg2;

output reg [31:0] systemcall_arg3;

reg [3:0] cur_state;

reg [3:0] next_state;

wire setup_finished;

assign setup_finished = read_buffer_cnt >= length;

always@(posedge clk)

begin

if (reset)

cur_state $<=$ STATE_reset;

else if (memory_controller_waitrequest)

cur_state $<=$ cur_state;

else

cur_state $<=$ next_state;

end

always@(*)

begin

next_state $=$ cur_state;

case(cur_state)

STATE_reset:

if (start)

next_state $=$ STATE_setup;

// Setup state -> read $>>$ write loop until the input is written to a buffer. Idea is to

// give the address of the buffer to the kernel module, which reads the buffer to determine

// the filename and opens it. BUT if bit30 of arg_0 is 1, that means the input bufer

$/ /$ is already in DDR SDRAM. If alraedy in DDR, simply hand off address to kernel module.

// This assumes that the caller provides PHYSICAL ADDRESS! This would be the case if

// the SW was compiled using AISLE or is modified to do virt to phys conversion before calling

// the accelerator.

STATE_setup:

if (setup_finished $==1^{\prime} b 1||$ arg_0[30] == 1'b1)

else

next_state $=$ STATE_started;

STATE_readpending:

next_state $=$ STATE_readpending;

next_state $=$ STATE_readpending_2; 


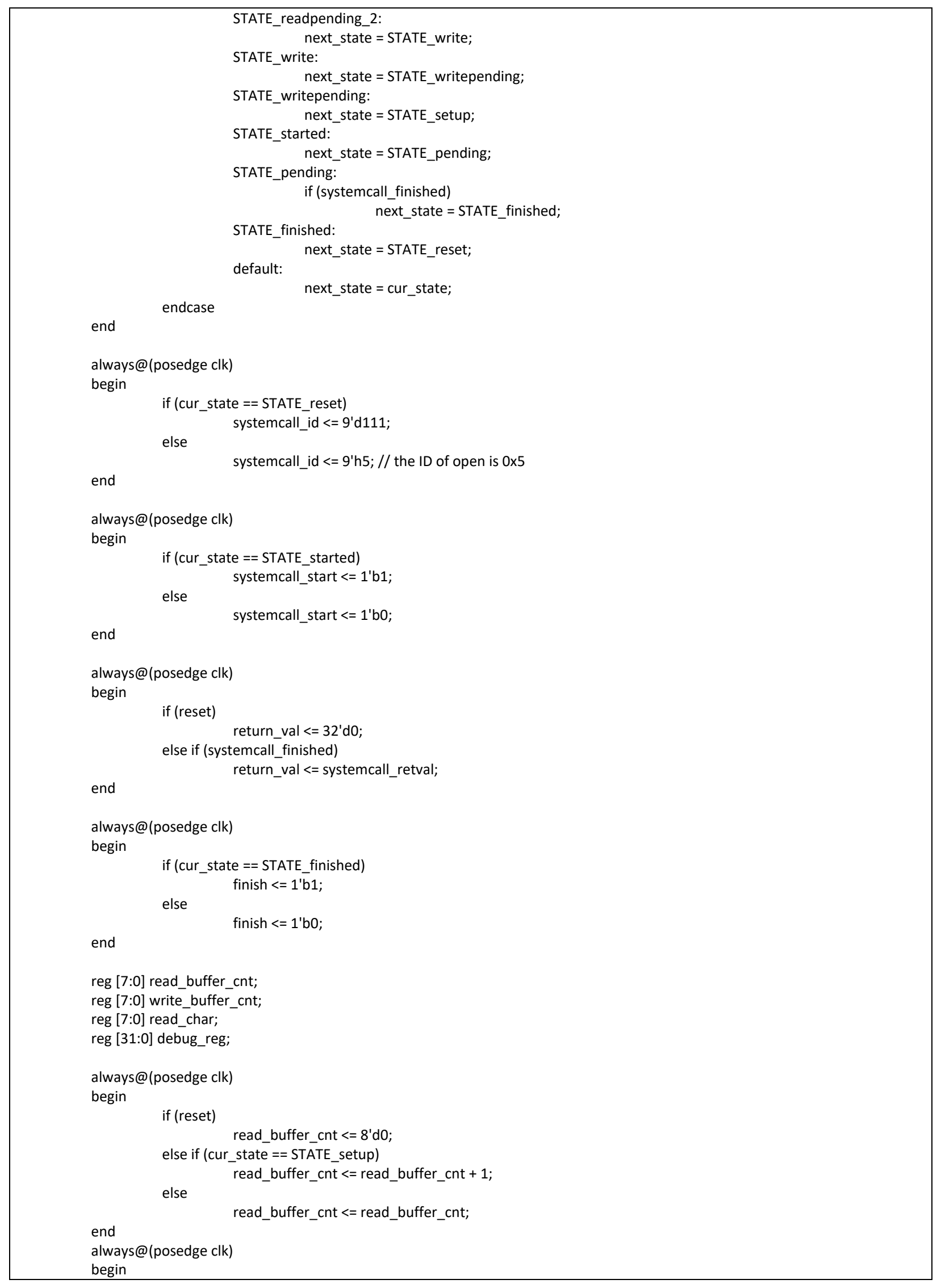




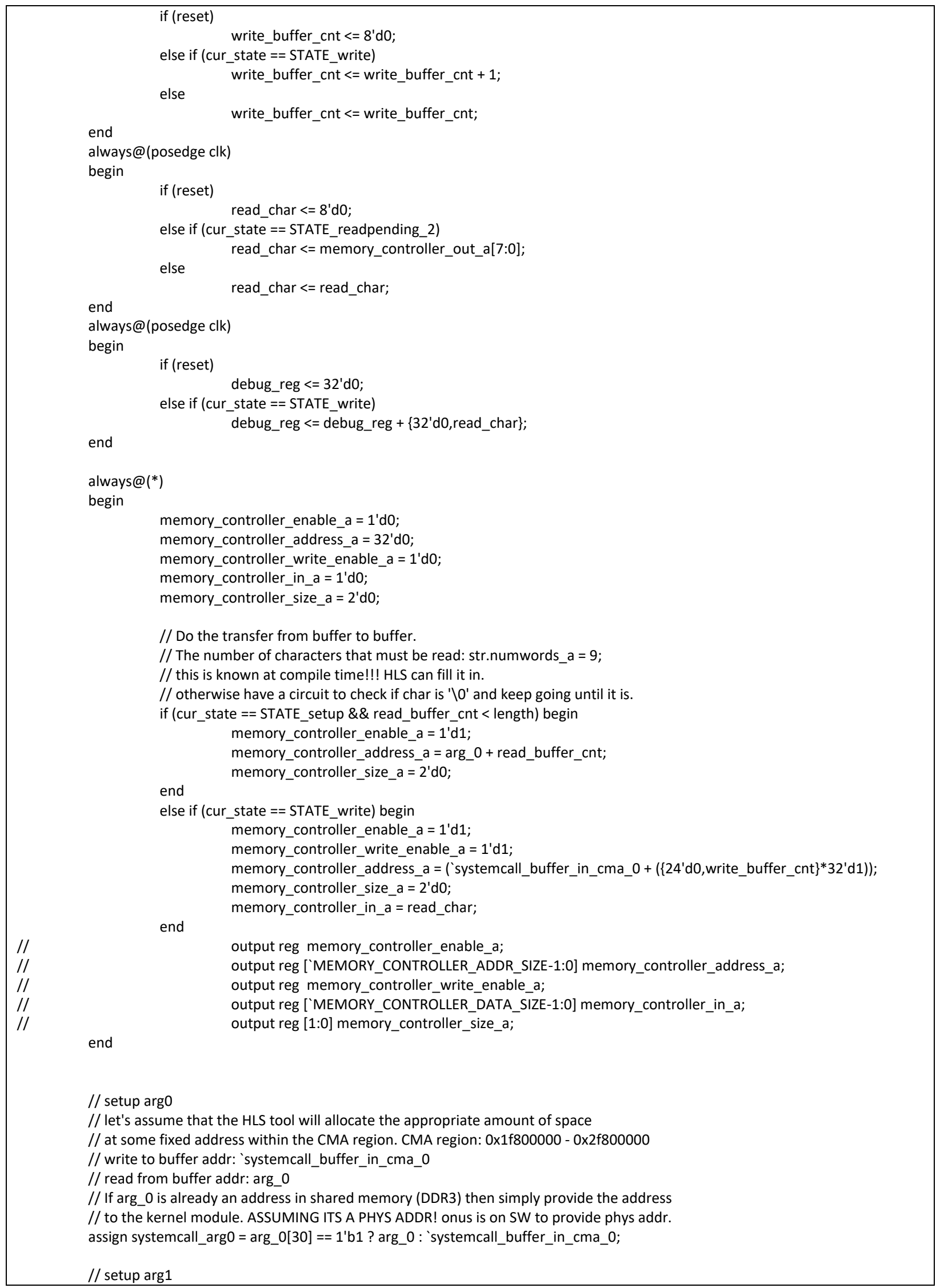




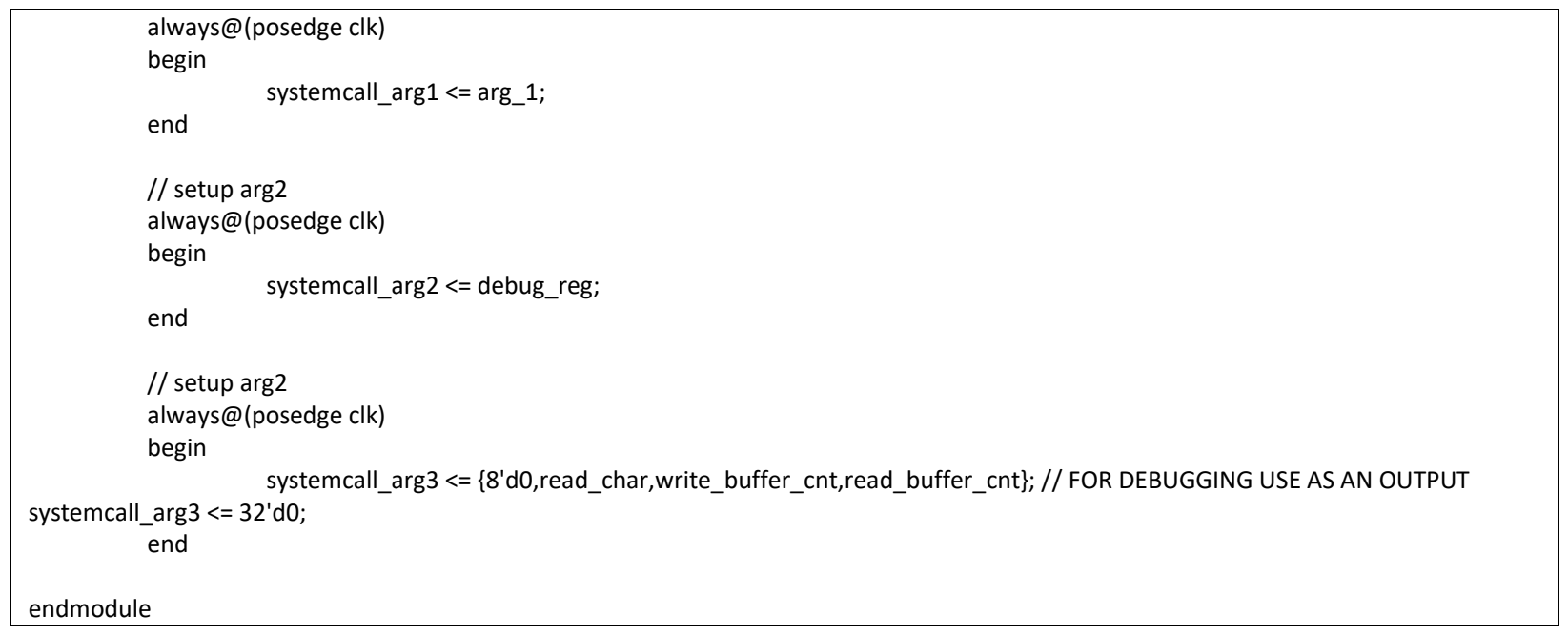

Figure 17 Verilog module that initiates the open() system call from the accelerator

\subsection{Verilog HDL Module that Initiates the Write System Call}

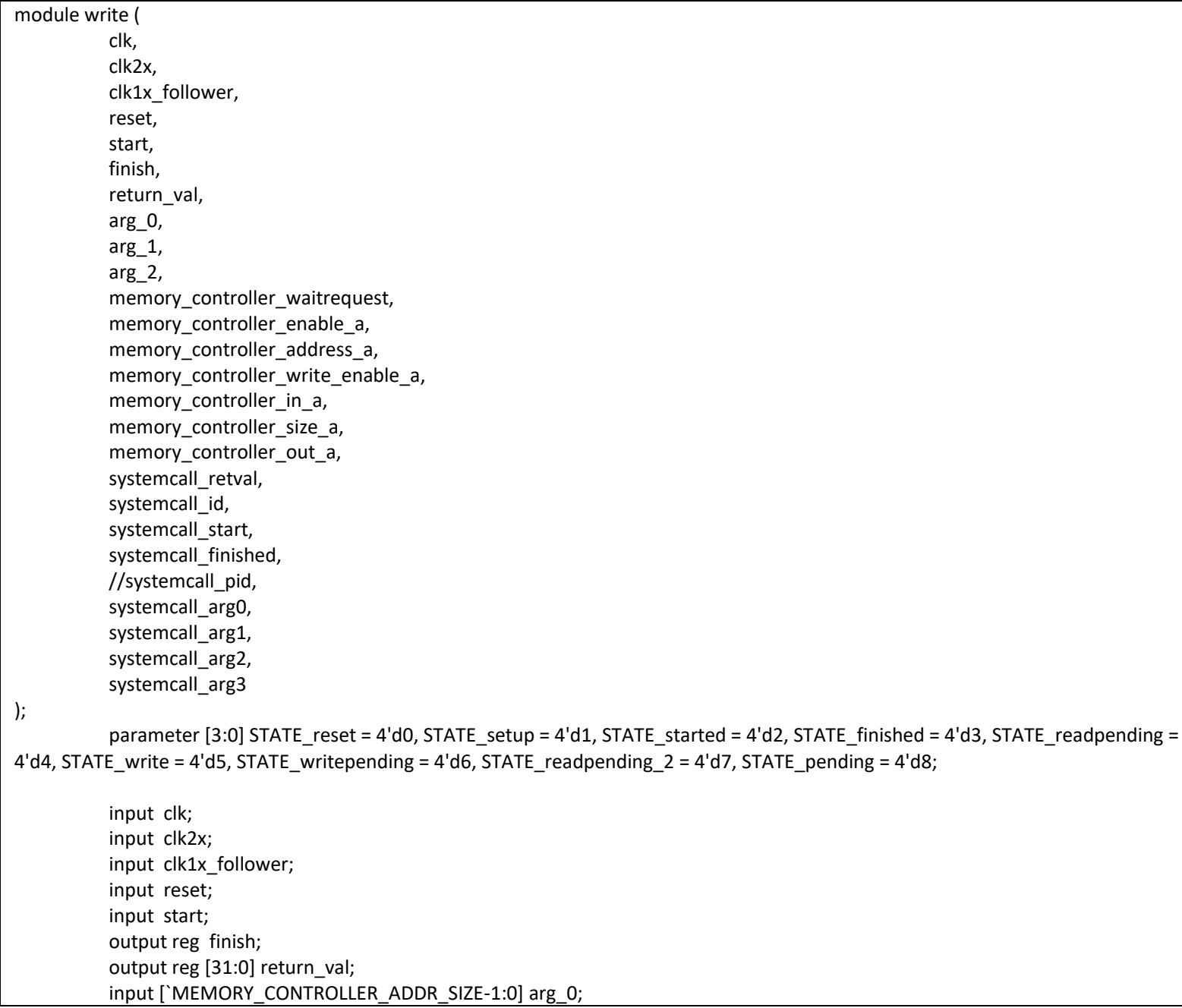




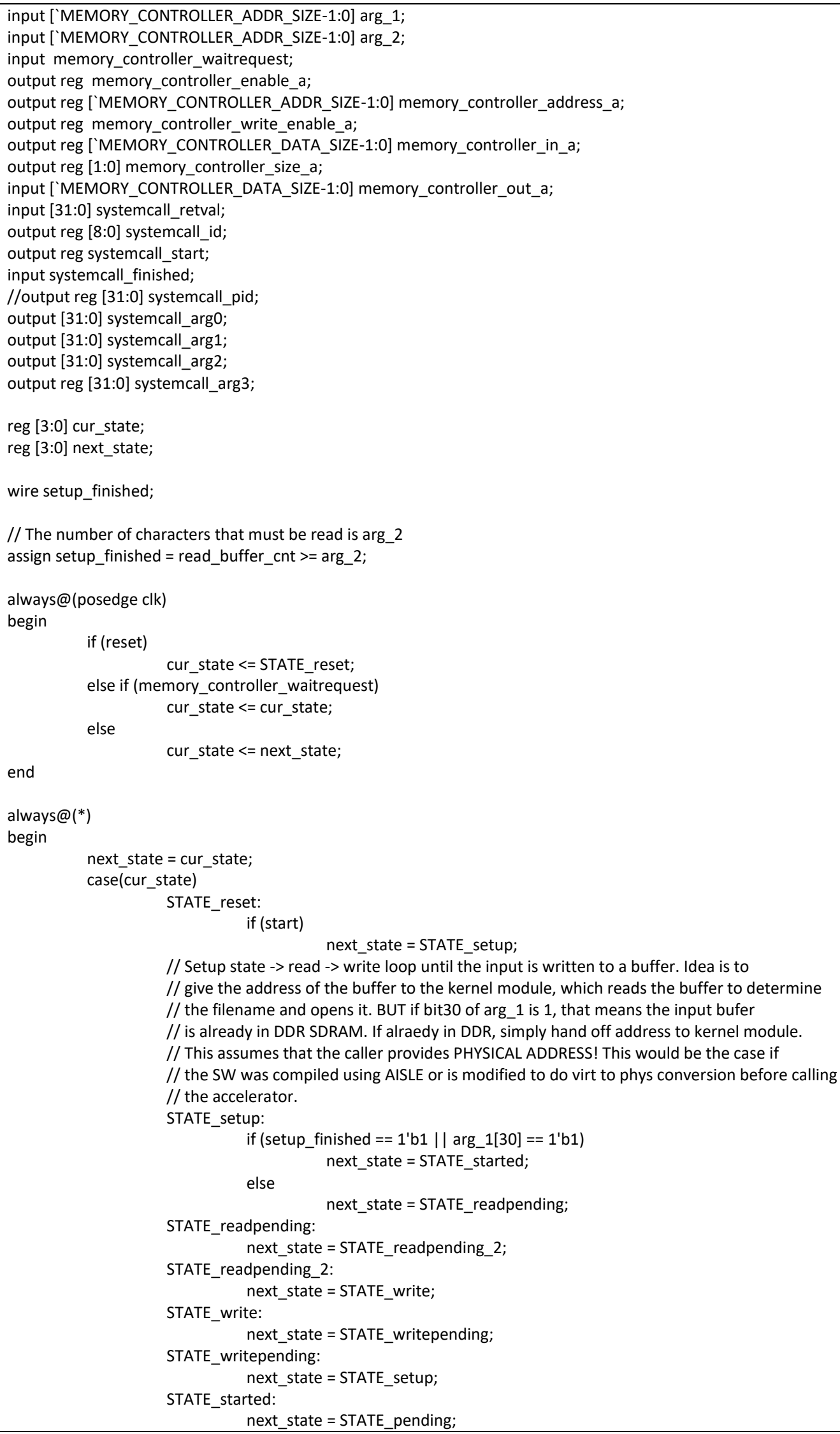









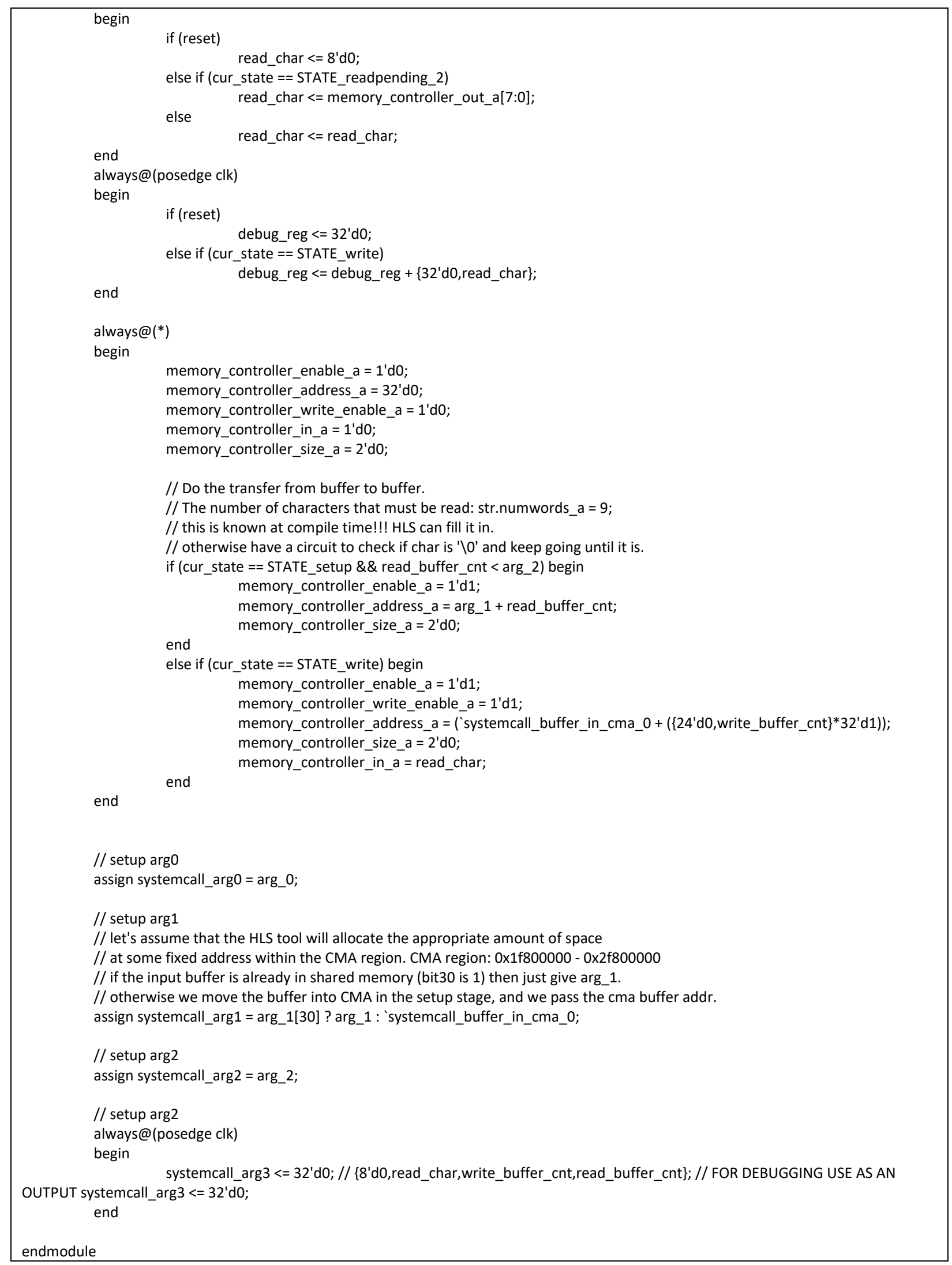

Figure 18 Verilog module that initiates the write() system call from the accelerator 


\subsection{Verilog HDL Module that Initiates the Close System Call}

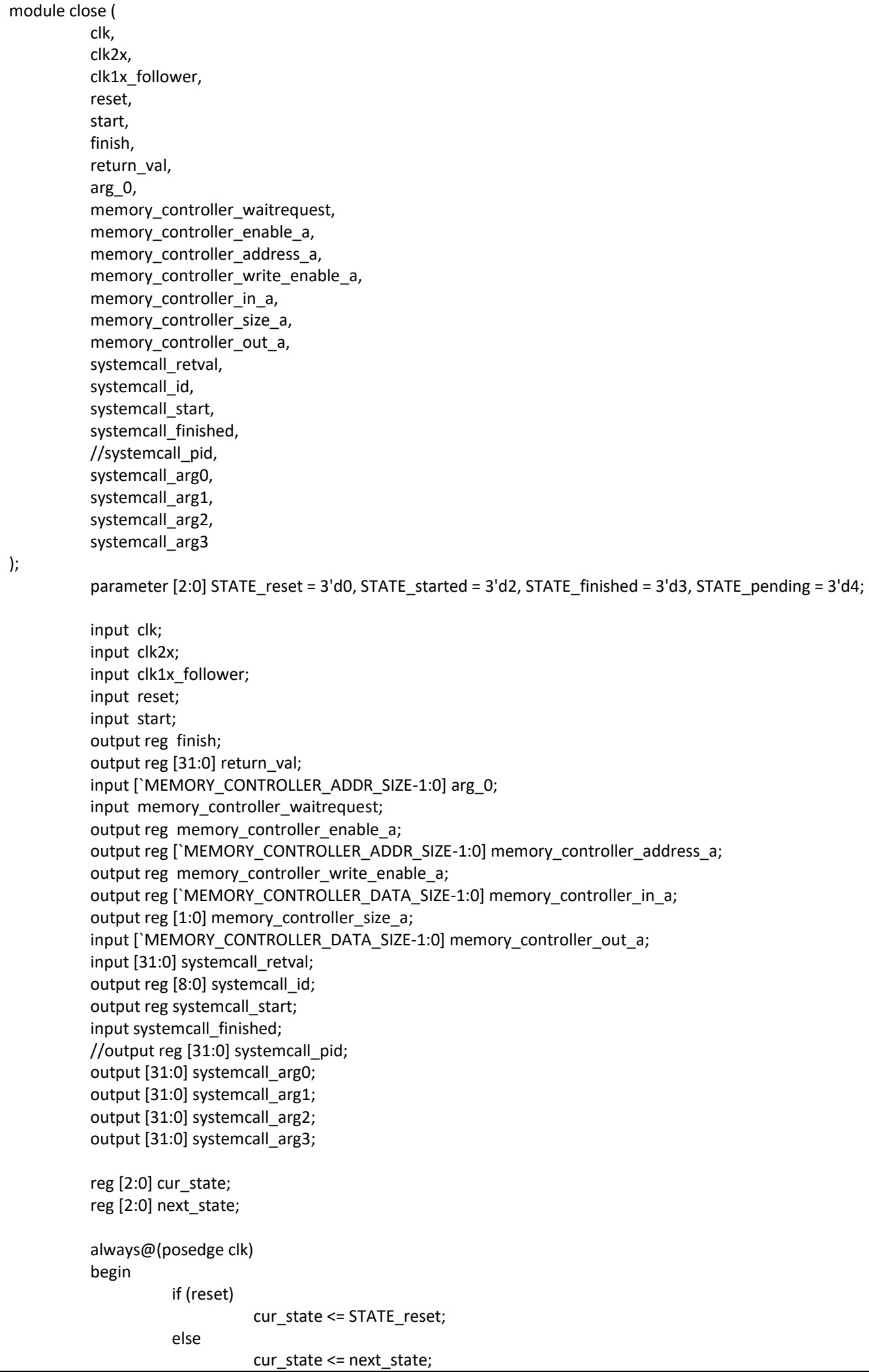




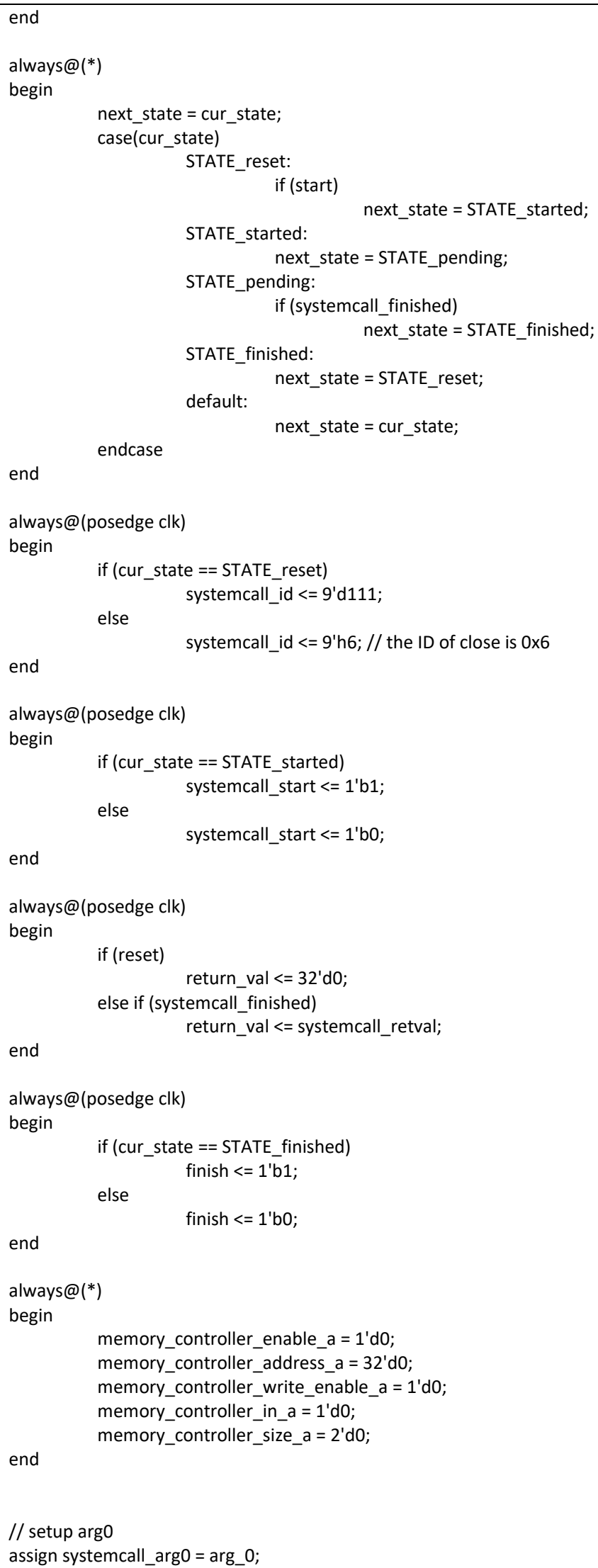




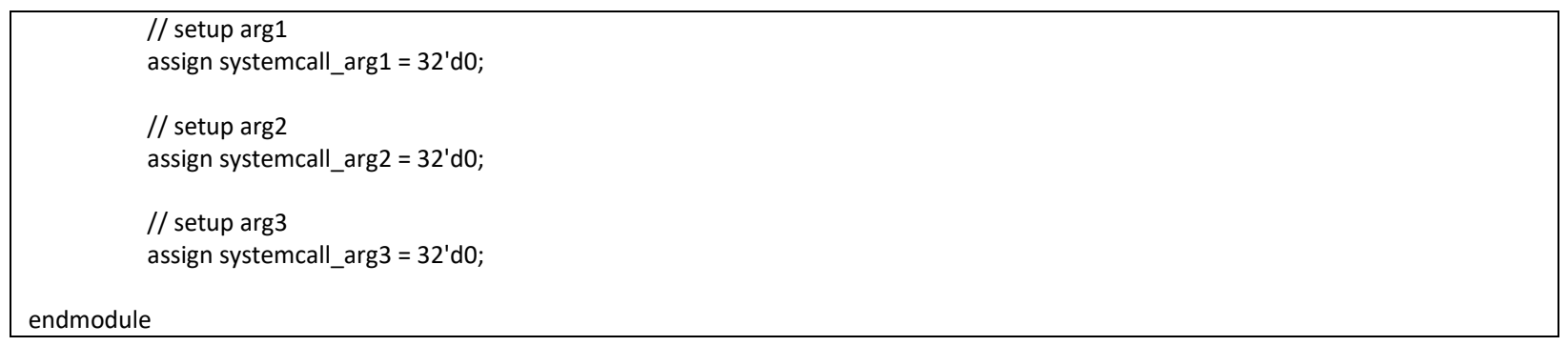

Figure 19 Verilog module that initiates the close() system call from the accelerator

\subsection{Linux Kernel Config File}

Omitted due to length. Contact author (kevin.nam at utoronto ca) for the code.

Figure 20 Linux kernel configuration settings for the custom 3.18 kernel used in the DE1-SoC

Linux distribution 


\section{References}

[1] I. Corporation, "Intel SoCs: When Architecture Matters," [Online]. Available: https://www.altera.com/products/soc/overview.html.

[2] X. Inc, "Expanding the All Programmable SoC Portfolio," [Online]. Available: https://www.xilinx.com/products/silicon-devices/soc.html.

[3] P. Gupta, "Xeon+FPGA Platform for the Data Center," 2015. [Online]. Available: https://www.ece.cmu.edu/ calcm/carl/lib/exe/fetch.php?media=carl15-gupta.pdf.

[4] I. Corporation, "SDK for OpenCL," [Online]. Available: https://www.altera.com/products/design-software/embedded-softwaredevelopers/opencl/overview.html.

[5] Xilinx Inc, "Vivado High-Level Synthesis," 2017. [Online]. Available: https://www.xilinx.com/products/design-tools/vivado/integration/esl-design.html.

[6] A. Canis, J. Choi, M. Aldham, V. Zhang, A. Kammoona, J. Anderson, S. Brown and T. Czajkowski, "LegUp: High-level synthesis for FPGA-based processor/accelerator systems," in ACM/SIGDA International Symposium on Field Programmable Gate Arrays (FPGA), Monterey, 2011.

[7] T. Team, "Intel Works To Gain An Upper Hand Over Nvidia In The Data Center Coprocessor Market," Forbes, 2016. [Online]. Available: 
https://www.forbes.com/sites/greatspeculations/2016/12/16/intel-works-to-gain-anupper-hand-over-nvidia-in-the-data-center-coprocessor-market/\#5c268d9b1178.

[8] Nallatech, "Choose the right FPGA Card for your Application," 2017. [Online]. Available: http://www.nallatech.com/solutions/fpga-cards/. [Accessed 5 May 2017].

[9] Terasic Technologies, "DE Main Boards," 2017. [Online]. Available: http://www.terasic.com.tw/cgi-bin/page/archive.pl?Language=English\&CategoryNo=163. [Accessed 5 May 2017].

[10] M. Kerrisk, "syscalls - Linux system calls," [Online]. Available: http://man7.org/linux/manpages/man2/syscalls.2.html.

[11] H. K.-H. So and R. Brodersen, "File system access from reconfigurable FPGA hardware processes in BORPH," in FPL, Heidelberg, 2008.

[12] Terasic Technologies Inc, "DE1-SoC User Manual," 2014.

[13] I. Corporation, "Qsys - System Integration Tool," [Online]. Available: https://www.altera.com/products/design-software/fpga-design/quartusprime/features/qts-qsys.html. [Accessed 193 2017].

[14] E. Lubbers and M. Platzner, "ReconOS: An RTOS Supporting Hard-and Software Threads," in FPL, Amsterdam, 2007. 
[15] J. Agron, W. Peck, E. Anderson, D. Andrews, E. Komp, R. Sass, F. Baijot and J. Stevens, "Run-Time Services for Hybrid CPU/FPGA Systems on Chip," in IEEE International RealTime Systems Symposium, Rio de Janeiro, 2006.

[16] D. B. Thomas, S. T. Fleming, G. A. Constantinides and D. R. Ghica, "Transparent linking of compiled software and synthesized hardware," in Design, Automation \& Test in Europe Conference \& Exhibition (DATE), Grenoble, 2015.

[17] H. K.-H. So, "BORPH: An Operating System for FPGA-Based Reconfigurable," EECS, University of California, Berkeley, 2007.

[18] IBM, "POWER8 Coherent Accelerator Processor Interface (CAPI)," [Online]. Available: https://www-304.ibm.com/webapp/set2/sas/f/capi/home.html.

[19] Microsoft, "Project Catapult," [Online]. Available: https://www.microsoft.com/enus/research/project/project-catapult/.

[20] A. E. Hindborg, P. Schleuniger, N. B. Jensen and S. Karlsson, "Hardware Realization of an FPGA Processor - Operating System Call Offload and," in Proceedings of the 2014 Conference on Design and Architectures for Signal and Image Processing (DASIP), Madrid, 2014. 